\title{
Pricing Currency Derivatives under the Benchmark Approach
}

\author{
November 8, 2014
}

\begin{abstract}
This paper considers the realistic modelling of derivative contracts on exchange rates. We propose a stochastic volatility model that recovers not only the typically observed implied volatility smiles and skews for short dated vanilla foreign exchange options but allows one also to price payoffs in foreign currencies, lower than possible under classical risk neutral pricing, in particular, for long dated derivatives. The main reason for this important feature is the strict supermartingale property of benchmarked savings accounts under the real world probability measure, which the calibrated parameters identify under the proposed model. Using a real dataset on vanilla option quotes, we calibrate our model on a triangle of currencies and find that the risk neutral approach fails for the calibrated model, while the benchmark approach still works.
\end{abstract}

KEYWORDS: Forex Market; Smile and Skew of Vanilla Options; 3/2 Stochastic Volatility Model; Strict Local Martingale; Model Calibration; Benchmark Approach.

\section{Introduction}

The globalization of the world economy has reached a significant level over recent decades. By exchanging goods and services the different economies exchange also their currencies. Consequently, the exchange rates between different currencies are key quantities in risk management and economic analysis. Since the recent financial crisis, investors tend to search for products, including foreign currency derivatives, with a long time horizon that are less sensitive to short-term market fluctuations and, potentially, are less expensive than current products.

There exists a wide literature on modelling the dynamics of foreign exchange (FX) rates. The majority of papers aims at capturing the stochasticity of the volatilities of exchange rates to recover typically observed derivative prices. Examples for this line of research are given by e.g. Beneder and Elkenbracht-Huizing (2003), Clark (2011), Castagna (2010), Bakshi et al. (2008).

Our main goal will be to provide a highly tractable FX-rate model under the real world probability measure, and to demonstrate how to price and hedge realistically short dated and long dated FX derivative contracts. Most importantly, it will turn out that the proposed model does not fit under the classical risk neutral approach. When the classical approach is still formally applied, it delivers more expensive prices than are possible to hedge under the real world pricing of the benchmark approach, see Platen and Heath (2010). The current paper seeks to construct an FX rate model that may lead beyond the classical risk neutral paradigm. This gives the freedom to capture features that are not present in the risk neutral models. By modeling purely under the real world probability measure and by indentifying the minimal possible prices, less expensive prices for long 
dated derivatives than the risk neutral ones become possible. The proposed model is aimed to be highly tractable, where we mean that we will favour a model structure where explicit or semiexplicit Fourier transforms for option prices exist that can be conveniently numerically inverted. Finally, we accept the fact that no model can ever shown to be the correct model. However, our fit shall provide confidence that the model represents a clear progress in modeling FX rates.

We exploit throughout the paper the following fact: When interpreting the MSCI total return world index as numéraire portfolio (NP) of a complete market model, it follows that the benchmarked US dollar (USD) savings account represents the Radon-Nikodym derivative for the putative risk neutral measure for the USD denomination of securities. Similarly, the benchmarked Euro (EUR) savings account can be interpreted as Radon-Nikodym derivative for the EUR market denomination. Under classical risk neutral assumptions both benchmarked savings accounts should be martingales. However, in both cases empirical evidence shows a systematic downward trend of the benchmarked savings accounts, see e.g. Platen and Heath (2010). This hints at the fact that these Radon-Nikodym derivatives may not be martingales and have to be potentially modelled as strict supermartingales in any advanced long-term FX model, as suggested in Platen and Heath (2010). We allow in this paper this possibility and let the data decide whether a risk neutral model may be sufficiently realistic. The current paper aims to be probably the first paper to present a stochastic volatility model that is calibrated to real market data without assuming that the risk neutral measure exists and still applies a coherent pricing method. Several authors (see e.g. Christensen and Larsen (2007), Delbaen and Schachermayer (1995), Hulley (2010), Karatzas and Kardaras (2007), Loewenstein and Willard (2000)) have investigated models where an equivalent martingale measure may fail to exist. In the absence of an equivalent martingale measure, many of the usual results of mathematical finance seem to break down. However, the concept of market completeness, namely the capability to replicate every contingent claim, must be kept distinct from the existence of an equivalent martingale measure. Indeed, the financial market may be viable and complete regardless of the existence of such measure (see Platen and Heath (2010)).

The model we propose is an advanced model that under the real world probability measure models realistically benchmarked savings accounts also over extreme long periods of time. Consequently, it recovers not only the typically observed implied volatility smiles and skews for short dated vanilla FX options, but allows one also to hedge long dated payoffs in foreign currencies for a lower price than possible under classical risk neutral pricing. As will become clear in the paper, the main reason for this important phenomenon is the strict supermartingale property of benchmarked savings accounts under the proposed model. Our new model reveals flexibility in capturing the typical symmetries that must be verified in any advanced multi-currency market model. For instance, this concerns the triangular relationship, which requires that products and ratios of exchange rates must also be exchange rates. This is introducing some constraints on the relations among the modelling processes. Moreover, we provide empirical evidence on the ability of this new framework to achieve pricing results consistent with real market prices, including the smile and skew effects in option prices. This results in a model that is able to simultaneously fit all the volatility surfaces, e.g. in the triangle Euro (EUR), US dollar (USD), and Japanese Yen (JPY). We obtain a framework to price consistently multi-asset claims by taking into account the skew of the three currency pairs, a very challenging task for any stochastic volatility model. In fact, a simultaneous calibration to all volatility smiles in the currency triangle is, in general, difficult to obtain, as a constant correlation does not provide enough flexibility to match the smile of the FX cross (see e.g. De Col et al. (2013)).

We will accomplish our goal by using a new multi-asset stochastic volatility model under the approach proposed in Heath and Platen (2006). Our model extends the minimal market model (MMM) introduced by Platen (2001) to a multifactor framework by adopting the $3 / 2$ model in- 
vestigated by Heston (1997), Platen (1997) and independently by Lewis (2000). The $3 / 2$ model assumes that the volatility factor is given by the inverse of a squared Bessel process. It has been already applied in different contexts, for instance by Ahn and Gao (1999), Spencer (2003), Carr and Sun (2007), Itkin and Carr (2009), Platen (1997), Chan and Platen (2011) and Drimus (2011). Despite its non affine nature, the $3 / 2$ specification is analytically tractable. This property provides the high tractability of the proposed model. The model also enjoys empirical support in the equity market, see e.g. Bakshi et al. (2006). Unlike the Heston model it leads to a variance that reverts quickly when it is high. This important fact is critical since it is empirically well observed in reality.

This paper is closely related to the literature on bubbles, see e.g. Heston et al. (2007), Jarrow et al. (2010), Jarrow and Protter (2010), and in particular Protter (2012) for a review article, to name but a few references. In this stream of literature, the situation in which the Radon-Nikodym derivative of the putative risk-neutral measure is a strict local martingale (that is supermartingale) is referred to as a money market bubble. In particular, Jarrow and Protter (2010) address a problem related to the one studied in this paper. In Section 2.3 we recall key concepts from the literature on bubbles, and highlight the differences between our contribution and the one by Jarrow and Protter (2010), who do not go beyond the classical risk neutral modelling.

The paper is organized as follows: In Section 2 we introduce our stochastic volatility model together with some basic properties. In particular, we explicitly determine the parameter set for which the model fits still under the classical risk neutral approach and the one where it does not admit any longer an equivalent risk neutral martingale measure. This description involves an inequality that can be easily checked on model parameters. In Section 3 we apply the benchmark approach in our context and provide a semi-closed form formula for the price of vanilla options. In Section 4 we calibrate the model on a dataset of real option prices. We find that the risk neutral methodology often fails, in the sense that the risk neutral probability measure does not exist for some currency denominations. Moreover, we find that the strict local martingality property of the benchmarked savings account cannot be neglected even for very short maturities, which is in line with theoretical results of Mijatović and Urusov (2012) and Bayraktar et al. (2012). This strongly emphasizes the motivation of this paper, which aims to bring realistic long term FX rates modelling beyond the risk neutral approach.

\section{The Model}

We introduce a filtered probability space $(\Omega, \mathcal{F}, \underline{\mathcal{F}}, \mathbb{P})$, with filtration $\underline{\mathcal{F}}=\left(\mathcal{F}_{t}\right)_{t \geq 0}$ satisfying the usual conditions. There are $N+1$ currencies in the model. The exchange rate between currencies $i$ and $j$ is denoted by $S^{i, j}, i, j=0, . ., N$. That is, $S^{i, j}(t)$ denotes the price of one unit of the $j$-th currency at time $t \in[0, T]$, when measured in units of the $i$-th currency. We refer to the currency with $i=0$ as the domestic currency, so that $S^{0, j}$ denotes the exchange rate for the $j$-th currency from the point of view of the domestic currency.

With respect to the $i$-th currency denomination of prices, $i=0, \ldots, N$, the $i$-th money-market account $B^{i}$ evolves, for simplicity, according to the constant risk free rate $r^{i}$, namely

$$
d B^{i}(t)=r^{i} B^{i}(t) d t
$$

We will now briefly recall some basic notions on the benchmark approach applied to the multicurrency market (for a complete survey of this approach we refer to Heath and Platen (2006) and Platen and Heath (2010)). We assume the existence of the Growth Optimal Portfolio (GOP), and will denote by $D^{i}(t)$ the GOP denominated in the $i$-th currency, $i=0, \ldots, N$. It can be shown 
that the dynamics of the GOP denominated in the $i$-th currency satisfies the following stochastic differential equation (SDE):

$$
\frac{d D^{i}(t)}{D^{i}(t)}=r^{i} d t+<\pi^{i}(t), \pi^{i}(t) d t+d \mathbf{Z}(t)>
$$

where $<.$, . $>$ denotes the scalar product on $\mathbb{R}^{d}$. $\mathbf{Z}$ is a $\mathbb{P}$-standard Brownian motion on $\mathbb{R}^{d}$ and the market prices of risk for the $i$-th currency denomination, $\pi^{i}$, are $\underline{\mathcal{F}}$-measurable. Benchmarked prices (i.e. prices denominated in terms of the GOP) are positive $\mathbb{P}$-local martingales (hence $\mathbb{P}$-supermartingales by Fatou's lemma). Since exchange rates can be expressed as ratios of two currency denominations of the GOP (i.e. $S^{i, j}(t)=D^{i}(t) / D^{j}(t)$ ) it follows that (see Heath and Platen (2006))

$$
\frac{d S^{i, j}(t)}{S^{i, j}(t)}=\left(r^{i}-r^{j}\right) d t+<\pi^{i}(t)-\pi^{j}(t), \pi^{i}(t) d t+d \mathbf{Z}(t)>
$$

Our aim is to propose a highly tractable model, which allows parameter choices whose benchmarked savings accounts are strict supermartingales. Therefore, in this paper we assume that the diffusion coefficient or $i$-th market price of risk vector $\pi^{i}(t)$ is a projection of some common $d$-dimensional volatility factor $\mathbf{V}(t)=\left(V_{1}(t), . ., V_{d}(t)\right)^{\top}$ along a direction parametrized by a constant vector $\mathbf{a}^{i} \in \mathbb{R}^{d}$ according to the following relation:

$$
\pi^{i}(t)=(\operatorname{Diag}(\mathbf{V}(t)))^{1 / 2} \mathbf{a}^{i}, \quad i=0, . ., N .
$$

Here $(\operatorname{Diag}(\mathbf{u}))^{1 / 2}$ denotes the diagonal matrix whose diagonal entries are the respective square roots of the components of the vector $\mathbf{u} \in \mathbb{R}^{d}$. Under this assumption, the dynamics of the GOP denominated in the $i$-th currency becomes

$$
\frac{d D^{i}(t)}{D^{i}(t)}=r^{i} d t+\left(\mathbf{a}^{i}\right)^{\top}(\operatorname{Diag}(\mathbf{V}(t))) \mathbf{a}^{i} d t+\left(\mathbf{a}^{i}\right)^{\top}(\operatorname{Diag}(\mathbf{V}(t)))^{1 / 2} d \mathbf{Z}(t) .
$$

Consequently, the dynamics of the exchange rate $S^{i, j}$ is given by

$$
\frac{d S^{i, j}(t)}{S^{i, j}(t)}=\left(r^{i}-r^{j}\right) d t+\left(\mathbf{a}^{i}-\mathbf{a}^{j}\right)^{\top}(\operatorname{Diag}(\mathbf{V}(t)))^{1 / 2}\left((\operatorname{Diag}(\mathbf{V}(t)))^{1 / 2} \mathbf{a}^{i} d t+d \mathbf{Z}(t)\right)
$$

The common stochastic factor $\mathbf{V}(t) \in \mathbb{R}^{d}$ is a vector whose components evolve according to the following dynamics:

$$
d V_{k}(t)=\kappa_{k} V_{k}(t)\left(\theta_{k}-V_{k}(t)\right) d t+\sigma_{k} V_{k}(t)^{3 / 2} d W_{k}(t), \quad k=1, . ., d ;
$$

where $\omega_{k}, \theta_{k}, \sigma_{k} \in \mathbb{R}_{+}, \kappa_{k}>-\sigma_{k}^{2} / 2$. Here $\mathbf{W}=\left(W_{1}, . ., W_{d}\right)^{\top}$ is a $\mathbb{P}$-standard Brownian motion correlated with $\mathbf{Z}$ through $d<W_{k}, Z_{k}>_{t}=\rho_{k} d t(k=1, . ., d)$ and $V_{k}(0)=v_{k} \in R^{+}$. The SDE (7) involves the power $3 / 2$ in the diffusion term and for this reason its solution is also called $3 / 2$ process, independently introduced in finance by Heston (1997), Platen (1997) and Lewis (2000). This process is obtained by taking the inverse of a square-root process of dimension strictly greater than 2. In fact, defining $X_{k}(t)=\frac{1}{V_{k}(t)}$, then

$$
d X_{k}(t)=\left(\kappa_{k}+\sigma_{k}^{2}-\kappa_{k} \theta_{k} X_{k}(t)\right) d t-\sigma_{k} \sqrt{X_{k}(t)} d W_{k}(t)
$$

and using Feller's explosion test (see e.g. Karatzas and Shreve (1991) Chapter 5, Th. 5.29), it follows that $V_{k}$ remains strictly positive and does not reach $+\infty$ in finite time. Note that, the stochastic volatility model obtained by choosing directly the solution of the SDE (8) as volatility factors is intimately related to a Heston (1997) model, where the Feller condition is systematically 
satisfied. However, it is important to note that for our model the parameters $\kappa_{k}$ and $\theta_{k}$ do no longer have the same interpretation and scaling as in the Heston model. In fact, the speed of linear mean reversion in the $3 / 2$ model is given by the one of $X_{k}$, which is the product $\kappa_{k} \theta_{k}(t)$. In particular, we find that the variance $V_{k}(t)$ will mean revert in (7) more quickly when it is high. This is a desirable empirically observed feature that is naturally expressed in the $3 / 2$ model and is not found e.g. in the Heston model.

For high tractability our model keeps the functional symmetry and triangular properties of the Heston-based version of De Col et al. (2013), that in turn takes inspiration of the original idea in Heath and Platen (2006). In particular, it is easily checked that the dynamics of the exchange rate in the $\mathrm{SDE}(6)$ satisfies the triangular relation. Namely, for all $t \geq 0$ and $i, j, l=0, . ., N$, the dynamics of a cross $d\left(S^{i, l} S^{l, j}\right)$, computed by applying Ito's rule to the product, gives the dynamics of a process that shares the same functional form of both $S^{i, l}$ and $S^{l, j}$ and is that of $S^{i, j}$. We emphasize that this functional symmetry property is fundamental in order to have a model that is consistent with a simultaneous fit of several implied volatility surfaces ${ }^{1}$.

\subsection{Strict Supermartingale Property}

In this subsection, we investigate the conditions under which the benchmarked savings accounts, $\hat{B}^{i}(t)=\frac{B^{i}(t)}{D^{i}(t)}$, are strict $\mathbb{P}$-local martingales, that is strict supermartingales, for all currencies $i=0, \ldots, N$. As argued in Platen and Heath (2010), for economic reasons, it is plausible to model benchmarked saving accounts as strict supermartingales. Furthermore, we note that $\hat{B}^{i}(t)$, after normalization to one at the initial time, corresponds to the candidate Radon-Nikodym derivative for the putative risk neutral measure of the $i$-th currency. Should $\hat{B}_{i}(t)$ be a strict $\mathbb{P}$-local martingale, we note that risk neutral pricing is not applicable, but real world pricing still is, as we are going to discuss in Subsection 2.2 .

From the dynamics of $\hat{B}^{i}(t)$ given by the SDE

$$
d \hat{B}^{i}(t)=-\hat{B}^{i}(t)\left(\mathbf{a}^{i}\right)^{\top} \operatorname{Diag}(\mathbf{V}(t))^{1 / 2} d \mathbf{Z}(t),
$$

it follows

$$
\hat{B}^{i}(t)=\hat{B}^{i}(0) \exp \left\{-\int_{0}^{t}\left(\mathbf{a}^{i}\right)^{\top} \operatorname{Diag}(\mathbf{V}(s))^{1 / 2} d \mathbf{Z}(s)-\frac{1}{2} \int_{0}^{t}\left(\mathbf{a}^{i}\right)^{\top} \operatorname{Diag}(\mathbf{V}(s)) \mathbf{a}^{i} d s\right\} .
$$

By the usual decomposition of the Brownian motion it follows that

$$
\begin{aligned}
\mathbb{E}\left[\hat{B}^{i}(t)\right] & =\hat{B}^{i}(0) \prod_{k=1}^{d} \mathbb{E}\left[\exp \left\{-a_{k}^{i} \int_{0}^{t} V_{k}^{1 / 2}(s) d Z_{k}(s)-\frac{1}{2}\left(a_{k}^{i}\right)^{2} \int_{0}^{t} V_{k}(s) d s\right\}\right] \\
& =\hat{B}^{i}(0) \prod_{k=1}^{d} \mathbb{E}\left[\exp \left\{-a_{k}^{i} \rho_{k} \int_{0}^{t} V_{k}^{1 / 2}(s) d W_{k}(s)-\frac{1}{2}\left(a_{k}^{i}\right)^{2} \rho_{k}^{2} \int_{0}^{t} V_{k}(s) d s\right\}\right] \\
& =\hat{B}^{i}(0) \prod_{k=1}^{d} \mathbb{E}\left[\xi_{k}^{i}(t)\right],
\end{aligned}
$$

\footnotetext{
${ }^{1}$ Note that if we start with a different specification of the model it may happen that taking the dynamics of the product of a pair breaks the structure of the dynamics of the cross, namely the model cannot be functionally symmetric. This is what happens for example to SABR or GARCH models, see the discussion in De Col et al. (2013) for more insights.
} 
where we define the exponential local martingale process $\xi_{k}^{i}=\left\{\xi_{k}^{i}(t), t \geq 0\right\}$ via

$$
\xi_{k}^{i}(t):=\exp \left\{-a_{k}^{i} \rho_{k} \int_{0}^{t} V_{k}^{1 / 2}(s) d W_{k}(s)-\frac{1}{2}\left(a_{k}^{i}\right)^{2} \rho_{k}^{2} \int_{0}^{t} V_{k}(s) d s\right\} .
$$

For a currency $i$, as soon as one of the processes $\xi_{k}^{i}$ is a strict local martingale for some $k=1, . ., d$, the process $\hat{B}^{i}$ is also a strict local martingale. Therefore, in such a case the risk neutral probability measure of the $i$-th currency denomination of prices will not exist. We now have the following result about the strict local martingality or strict supermartingale property of $\xi_{k}^{i}$.

Theorem 1. Given $i=0, . ., N$ and $k=1, . ., d$, the process $\xi_{k}^{i}$ given by equation (9) is a $\mathbb{P}$-strict local martingale if and only if

$$
\frac{\kappa_{k}}{\sigma_{k}}+\frac{\sigma_{k}}{2}<-a_{k}^{i} \rho_{k} .
$$

Moreover, condition (10) is necessary and sufficient for $\xi_{k}^{i}$ to be a strict local martingale on the time interval $[0, T]$ for any fixed $T \in(0, \infty)$.

Proof. See Appendix A.

Remark 1. The inequality (10) has been independently found by Drimus (2011) in a single-factor risk neutral framework, where he imposed some parameter restrictions on the model in order to ensure the risk neutral approach (that is, (10) is not satisfied).

Remark 2. In De Col et al. (2013) the authors employ a multi Heston-based version of the model, where the volatility factors evolve according to the following dynamics:

$$
d V_{k}(t)=\kappa_{k}\left(\theta_{k}-V_{k}(t)\right) d t+\sigma_{k}\left(V_{k}(t)\right)^{1 / 2} d W_{k}(t), \quad k=1, \ldots, d .
$$

When calibrating their model, De Col et al. (2013) found that the Feller condition is systematically violated (a usual stylized fact in the Heston stochastic volatility models, see e.g. Da Fonseca and Grasselli (2011)), which leads to inconsistency with the strict positivity of the volatility factors and other uncomfortable effects like moment explosions. Nevertheless, we point out that even if $V_{k}$ can reach 0 , the process $\hat{B}^{i}(t)=\frac{B^{i}(t)}{D^{i}(t)}$ remains always a martingale with the same specification (4) for the market price of risk, so that risk neutral pricing is always applicable in the Heston-like framework. This result is in line with observations in Andersen and Piterbarg (2007). To break free from the risk neutral assumptions, we employ the $3 / 2$ model, for which $\hat{B}^{i}$ can be also a strict local martingale. Moreover, in the 3/2 model the volatility factors never reach zero since they are inverses of square-root processes of dimension strictly greater than 2 and there are economic and empirical reasons that support this type of model, see Platen and Rendek (2012b).

Remark 3. When using the fact that $\kappa_{k}>-\sigma_{k}^{2} / 2$, which ensures that $X_{k}$ does not reach zero, we find that we have explosion of $\tilde{V}_{k}(t)$ as soon as $-a_{k}^{i} \rho_{k}>\frac{\kappa_{k}}{\sigma_{k}}+\frac{\sigma_{k}}{2}>0$. This means that the explosion is related to some negative correlation between $D^{i}(t)$ and $\tilde{V}_{k}(t)$. The GOP can be interpreted as a well-diversified equity index, see Platen and Rendek (2012a), and should exhibit the well-observed leverage effect. To achieve this the above model can most likely only become realistic when $\tilde{V}_{k}$ explodes, and hence risk neutral pricing will not be applicable.

\subsection{Real World Pricing}

We focus on a particular currency denomination, say the domestic one (corresponding to $i=0$ ), and recall the real world pricing formula, see e.g. Platen and Heath (2010). We fix a maturity date $T$ of a contingent claim with nonnegative $\mathcal{F}_{T}$-measurable payoff $H$ in domestic currency. If 
$\mathbb{E}\left[\frac{H}{D^{0}(T)}\right]<+\infty$, the real world pricing formula defines the price process of the claim $H$ at time $t \in[0, T]$ by

$$
p(t):=D^{0}(t) \mathbb{E}_{t}\left[\frac{H}{D^{0}(T)}\right],
$$

where $\mathbb{E}_{t}[]=.\mathbb{E}\left[. \mid \mathcal{F}_{t}\right]$. Note that the benchmarked price $\hat{p}(t)=p(t) / D^{0}(t)$ forms a martingale, since $\hat{p}(T)=H / D^{0}(T)$. Since all nonnegative self-financing benchmarked portfolios are supermartingales, the real world pricing formula provides the minimal possible price process. It has been shown in Platen and Heath (2010) in the case when a risk neutral probability measure exists, that $\hat{B}^{0}$ forms a martingale, and the real world pricing formula gives exactly the same prices as the risk neutral pricing formula. In this sense our approach generalizes the classical risk neutral one, and we can let the data decide whether the risk neutral approach is suitable or needs to be generalized.

We consider the pricing problem of a European call option on the exchange rate $S^{1,0}(T)=$ $D^{1}(T) / D^{0}(T)$, which can also be thought of as the ratio between the GOP denominated in the foreign currency $i=1$ and in the domestic currency $i=0$. The payoff in the domestic currency $i=0$ of a call with strike $K$ in the currency $i=1$ and maturity $T>0$ can be written as

$$
H=S^{0,1}(T)\left(S^{1,0}(T)-K\right)^{+}=\frac{D^{0}(T)}{D^{1}(T)}\left(\frac{D^{1}(T)}{D^{0}(T)}-K\right)^{+}=1-\min \left(K \frac{D^{0}(T)}{D^{1}(T)}, 1\right) .
$$

According to the real world pricing formula (11) applied to the domestic currency $i=0$, we are then led to compute for the benchmarked call option the following quantities

$$
\mathbb{E}_{t}\left[S^{0,1}(T)\left(S^{1,0}(T)-K\right)^{+} \frac{1}{D^{0}(T)}\right]=\mathbb{E}_{t}\left[\frac{1}{D^{0}(T)}\right]-\mathbb{E}_{t}\left[\min \left(\frac{K}{D^{1}(T)}, \frac{1}{D^{0}(T)}\right)\right] .
$$

We turn now our attention to the pricing of put options. Having derived the pricing formulas for call options and zero-coupon bonds, we can employ the fair put-call parity (see Platen and Heath (2010)) to price put options, thus, obtaining the following equality for the corresponding benchmarked put

$$
\begin{aligned}
\mathbb{E}_{t}\left[S^{0,1}(T)\left(K-S^{1,0}(T)\right)^{+} \frac{1}{D^{0}(T)}\right]= & \mathbb{E}_{t}\left[S^{0,1}(T)\left(S^{1,0}(T)-K\right)^{+} \frac{1}{D^{0}(T)}\right] \\
& -\mathbb{E}_{t}\left[\frac{1}{D^{0}(T)}\right]+K \mathbb{E}_{t}\left[\frac{1}{D^{1}(T)}\right] \\
& =K \mathbb{E}_{t}\left[\frac{1}{D^{1}(T)}\right]-\mathbb{E}_{t}\left[\min \left(\frac{K}{D^{1}(T)}, \frac{1}{D^{0}(T)}\right)\right] .
\end{aligned}
$$

Of course, if both $\hat{B}^{0}$ and $\hat{B}^{1}$ were martingales, then the above equality would reduce to the standard put-call parity.

\subsection{Connections with the Literature on Bubbles}

Since the appearence of strict local martingales is critical for our model, we briefly recall some key concepts from the literature on bubbles. In Heston et al. (2007), the authors define a money market bubble as the situation in which the money market account can be replicated at a fixed future time using a self-financing trading strategy whose setup cost is lower than the current value of the money market account. Hence in our model, a money market bubble exists if and only if the benchmarked savings account follows a strict local martingale that is a supermartingale. In our setup, condition 
(10) is a necessary and sufficient condition for the existence of a money market bubble with respect to currency $i$. This means that detecting money market bubbles is equivalent to detecting strict local martingales in the benchmarked savings account processes. We furthermore remark that if our model rules out money market bubbles, we can then investigate if under the risk neutral measure the discounted well-diversified index follows a strict local martingale. Should the latter be the case, one has detected an asset pricing bubble, using the terminology from Heston et al. (2007).

In Jarrow and Protter (2010), the authors investigate the existence of foreign currency bubbles. In particular, they study the existence of such bubbles in a nominal economy and in a real economy. Unlike in our setting, the authors assume the existence of an equivalent martingale measure and, consequently, investigate the existence of asset pricing bubbles. Regarding the nominal economy, having assumed the existence of an equivalent martingale measure, an asset pricing bubble exists if the price process of the foreign savings account, expressed in units of the domestic currency, follows a strict local martingale under this measure. Furthermore, an exchange rate exhibits a bubble if the ratio of the foreign savings account expressed in units of domestic currency, and the domestic savings account follows a strict local martingale. As Jarrow and Protter (2010) emphasize, a necessary consequence of this approach is that the existence of bubbles, i.e. strict local martingales, crucially depends on the equivalent martingale measure chosen and the corresponding numéraire. Choosing a different currency as numéraire and hence a different equivalent martingale measure impacts the characterization of bubbles.

Our approach is markedly different from Jarrow and Protter (2010), as we do not assume the existence of an equivalent martingale measure. This has the following two important benefits:

- Though we model a nominal economy, our detection of strict local martingales, i.e. money market bubbles, does not depend on the particular choice of the equivalent martingale measure and numéraire.

- In our analysis, exchange rates are defined as ratios of the GOP. We exclusively deal with the real world measure for which the GOP is the numéraire.

\section{Call Pricing in the Multivariate 3/2 Model}

The 3/2 stochastic volatility model does not belong to the class of affine processes (see Duffie et al. (2003)). Nevertheless, it is still highly tractable since it is possible to perform the Fourier analysis in order to price options through the FFT methodology as for the affine Heston (1993) model. In fact, the key point is that the moment generating function of log prices for the $3 / 2$ stochastic volatility model is known in closed form and it involves special functions (the hypergeometric confluent and incomplete Gamma functions) that can be easily implemented.

Let us first introduce for $j=0,1$ the $\log$ price $Y^{j}(t)=\log \left(D^{j}(t)\right)$ of the GOP $D^{j}(t)$, which satisfies the SDE

$$
d Y^{j}(t)=\left(r^{j}+\frac{1}{2}\left(\mathbf{a}^{j}\right)^{\top} \operatorname{Diag}(\mathbf{V}(t))^{-1} \mathbf{a}^{j}\right) d t+\left(\mathbf{a}^{j}\right)^{\top} \operatorname{Diag}(\mathbf{V}(t))^{-1 / 2} d \mathbf{Z}(t) .
$$

Then, using the notation $\mathbf{Y}(T):=\left(Y^{0}(T), Y^{1}(T)\right)^{\top}$, the price at time $t \leq T$ of the call option in domestic currency can be decomposed as

$$
\begin{aligned}
\text { Call }_{t} & =D^{0}(t) \mathbb{E}_{t}\left[\left(\frac{D^{1}(T)}{D^{0}(T)}-K\right)^{+} \frac{1}{D^{1}(T)}\right] \\
& =D^{0}(t)\left(\mathbb{E}_{t}\left[e^{-Y^{0}(T)}\right]-\mathbb{E}_{t}\left[F\left(Y^{0}(T), Y^{1}(T)\right)\right]\right),
\end{aligned}
$$


where for $\mathbf{y}=\left(y^{0}, y^{1}\right)^{\top} \in \mathbb{R}^{2}$ we have

$$
F(\mathbf{y})=F\left(y^{0}, y^{1}\right)=\min \left(\exp \left(-y^{0}\right), K \exp \left(-y^{1}\right)\right) .
$$

The first term on the right hand side of (13) is already a Laplace transform and it will be obtained analytically in the sequel. Let us focus on the second term: Using standard arguments, see e.g. Da Fonseca et al. (2007), we obtain

$$
\begin{aligned}
\mathbb{E}_{t}\left[F\left(Y^{0}(T), Y^{1}(T)\right)\right] & =\frac{1}{(2 \pi)^{2}} \mathbb{E}_{t}\left[\int_{\mathcal{Z}}\left(\int_{\mathbb{R}^{2}} e^{i\langle\boldsymbol{\lambda}, \mathbf{y}\rangle} F(\mathbf{y}) d \mathbf{y}\right) e^{-\mathrm{i}\langle\boldsymbol{\lambda}, \mathbf{Y}(T)\rangle} d \boldsymbol{\lambda}\right] \\
& =\frac{1}{(2 \pi)^{2}} \int_{\mathcal{Z}} \hat{F}(\boldsymbol{\lambda}) \Psi_{t}(-\mathbf{i} \boldsymbol{\lambda}) d \boldsymbol{\lambda},
\end{aligned}
$$

where $i=\sqrt{-1}$. Here $\langle\cdot, \cdot\rangle$ denotes the scalar product in $\mathbb{C}^{2}, \boldsymbol{\lambda}=\left(\lambda^{0}, \lambda^{1}\right)^{\top} \in \mathbb{C}^{2}$ and $\mathcal{Z}$ denotes the admissible domain of the Fourier transform of the payoff function $F$, which is defined as

$$
\hat{F}\left(\lambda^{0}, \lambda^{1}\right)=\int_{\mathbb{R}^{2}} e^{\mathrm{i} \lambda^{0} y^{0}+\mathfrak{i} \lambda^{1} y^{1}} \min \left(e^{-y^{0}}, K e^{-y^{1}}\right) d y^{0} d y^{1} .
$$

The function $\Psi_{t}$ is the two-dimensional (conditional) complex Fourier transform of the log GOP prices under the two currencies, that is

$$
\Psi_{t}(i \boldsymbol{\lambda})=\mathbb{E}_{t}[\exp (i\langle\boldsymbol{\lambda}, \mathbf{Y}(T)\rangle)] .
$$

Formula (14) states that the price can be computed by integrating numerically the product of two Fourier transforms: The first one, $\hat{F}$, depends on the payoff and is model independent. The second one, $\Psi_{t}$, depends on the model and is payoff independent.

\subsection{The Fourier Transform of the Payoff Function}

We first compute the Fourier transform $\hat{F}$ of the payoff function, together with its admissible domain, $\mathcal{Z}$.

Proposition 1. The Fourier transform of $F\left(y^{0}, y^{1}\right)=\min \left(e^{-y^{0}}, K e^{-y^{1}},\right)$ is given by

$$
\hat{F}\left(\lambda^{0}, \lambda^{1}\right)=\frac{K^{1-i \lambda^{0}}}{\lambda^{0}\left(\lambda^{0}-1\right)} 2 \pi \delta_{0}\left(\operatorname{Re}\left(\lambda^{0}\right)+\operatorname{Re}\left(\lambda^{1}\right)\right),
$$

where $\delta_{0}$ denotes the Dirac measure concentrated at 0 and the admissible domain is given by

$$
\mathcal{Z}=\left\{\lambda^{0} \in \mathbb{C}, \lambda^{1} \in \mathbb{C}: \operatorname{Im}\left(\lambda^{0}\right)+\operatorname{Im}\left(\lambda^{1}\right)=-1,-1<\operatorname{Im}\left(\lambda^{0}\right)<0\right\} .
$$

Proof. See Appendix A.

\subsection{The Characteristic Function of Log Prices}

We now compute the second transform, $\Psi_{t}$ :

$$
\begin{aligned}
\Psi_{t}(\mathbf{i} \boldsymbol{\lambda}) & \left.=\mathbb{E}_{t}[\exp (i \mathbf{i}, \mathbf{Y}(T)\rangle)\right] \\
& =f(t, \boldsymbol{\lambda}, \mathbf{y}, \mathbf{V}),
\end{aligned}
$$

where $\boldsymbol{\lambda}=\left(\lambda^{0}, \lambda^{1}\right), \mathbf{Y}(t)=\mathbf{y}=\left(y^{0}, y^{1}\right)^{\top}$ and $\mathbf{V}(t)=\mathbf{V}$. In addition, we are going to show that the characteristic function $\Psi_{t}$ is regular on $\mathcal{Z}$, that is it does not contain discontinuities on the integration region of formula (14). 
Proposition 2. Let $f$ be given by equation (19). Then

$$
f(t, \boldsymbol{\lambda}, \mathbf{y}, \mathbf{V})=\exp \left(i\langle\boldsymbol{\lambda}, \mathbf{y}\rangle+i(T-t)\left(r^{0} \lambda^{0}+r^{1} \lambda^{1}\right)\right) \prod_{k=1}^{d} u_{k}\left(\frac{2 \kappa_{k} \theta_{k}}{\sigma^{2} V_{k}}, \kappa_{k} \theta_{k}(T-t)\right),
$$

where

$$
u_{k}(x, t)=\frac{\Gamma\left(\beta_{k}-\alpha_{k}\right)}{\Gamma\left(\beta_{k}\right)}(X(x, t))^{\alpha_{k}} M\left(\alpha_{k}, \beta_{k},-X(x, t)\right)
$$

with

$$
\begin{aligned}
X(x, t) & =\frac{x}{\exp (t)-1} \\
\beta_{k} & =1+2 \delta_{k} ; \\
\delta_{k} & =\sqrt{\mu_{k}^{2}+\frac{2 c_{k}}{\sigma_{k}^{2}}} \\
\mu_{k} & =\frac{1}{2}\left(1+\frac{2 \kappa_{k}}{\sigma_{k}^{2}}-\frac{2 i \rho_{k}\left\langle\mathbf{a}_{\mathbf{k}}, \boldsymbol{\lambda}\right\rangle}{\sigma_{k}}\right) \\
\alpha_{k} & =\beta_{k}+\nu_{k}-1 \\
\nu_{k} & =-\mu_{k}-\delta_{k} ; \\
c_{k} & =-\left(\frac{i}{2} \sum_{j=0}^{1}\left(a_{k}^{j}\right)^{2} \lambda^{j}-\frac{1}{2}\left\langle\mathbf{a}_{\mathbf{k}}, \boldsymbol{\lambda}\right\rangle^{2}\right) \\
\mathbf{a}_{\mathbf{k}} & =\left(a_{k}^{0}, a_{k}^{1}\right)^{\top}
\end{aligned}
$$

and where $\Gamma$ is the (complex valued) Gamma function and $M$ denotes the hypergeometric confluent function (see e.g. Abramowitz and Stegun (1970)). Moreover, the solution $f(t, \boldsymbol{\lambda}, \mathbf{y}, \mathbf{V})$ does not contain discontinuities on the integration region $\mathcal{Z}$ of formula (14).

Proof. See Appendix A.

\subsection{Strict Local Martingality of the Price Deflator}

Let us now discuss when benchmarked savings accounts are strict local martingales, that is, strict supermartingales. If we compute the characteristic function (19) with $\lambda^{0}=i$ and $\lambda^{1}=0$, then we obtain the first term in the call price in (13), that is

$$
\begin{aligned}
\Psi_{t}(\mathrm{i}, 0) & =\mathbb{E}_{t}\left[e^{-Y^{0}(T)}\right] \\
& =\mathbb{E}_{t}\left[\frac{1}{D^{0}(T)}\right] .
\end{aligned}
$$

We recall from relation (10) in Subsection 2.1, that $\frac{B^{0}(t)}{D^{0}(t)}$ is a strict local martingale, if and only if there exists $k \in\{1, \ldots, d\}$, such that

$$
\frac{\kappa_{k}}{\sigma_{k}}+\frac{\sigma_{k}}{2}<-a_{k}^{0} \rho_{k}
$$

If we replace $\lambda^{0}=i$ and $\lambda^{1}=0$ in the solution given by Proposition 2, we get $\tilde{c}_{k}=0$ and $\delta=\left|\mu_{k}\right|$. Since

$$
\mu_{k}=\frac{1}{2}\left(1+\hat{\theta}_{k}\right)
$$


we get that

$$
\mu_{k}<0 \Leftrightarrow \frac{\sigma_{k}}{2}+\frac{\kappa_{k}}{\sigma_{k}}<-\rho_{k} a_{k}^{0}
$$

where the latter condition is the one appearing in (10). Assuming that condition (10) is satisfied, it follows $\nu_{k}=0, \delta_{k}=-\mu_{k}$ and $\alpha_{k}=\beta_{k}-1$. Consequently, using the properties of the confluent hypergeometric function, one has

$$
u_{k}\left(y_{k}, t_{k}\right)=\frac{\Gamma\left(\beta_{k}-1\right)-\Gamma\left(\beta_{k}-1, X\left(y_{k}, t_{k}\right)\right)}{\Gamma\left(\beta_{k}-1\right)}<1
$$

where $\Gamma(.,$.$) denotes the incomplete Gamma function (see e.g. Abramowitz and Stegun (1970)).$ Alternatively, if condition (10) is not satisfied, then $\mu_{k}=\delta_{k}, \nu_{k}=-2 \delta_{k}$ and $\alpha_{k}=0$. In this case we obtain from the properties of the confluent hypergeometric function

$$
u_{k}\left(y_{k}, t_{k}\right)=1 \text {. }
$$

Since

$$
\mathbb{E}_{t}\left[\frac{1}{D^{0}(T)}\right]=\frac{e^{-r_{0}(T-t)}}{D^{0}(t)} \prod_{k=1}^{d} u_{k}\left(y_{k}, t_{k}\right),
$$

it follows that

$$
\mathbb{E}_{t}\left[\frac{1}{D^{0}(T)}\right]<\frac{e^{-r_{0}(T-t)}}{D^{0}(t)}
$$

that is, $\frac{B^{0}(t)}{D^{0}(t)}$ is a strict local martingale, if and only if there exists a $k \in\{1, \ldots d\}$ so that

$$
\frac{\sigma_{k}}{2}+\frac{\kappa_{k}}{\sigma_{k}}<-\rho_{k} a_{k}^{0}
$$

which is consistent with Theorem 1.

\section{Model Calibration on Real Data}

There are at least two ways of calibrating and fitting the model. The first one, discussed in this paper, looks forward and uses derivatives data, which give a reflection of the market's view about the future evolution of the underlyings. The second one uses historical observations of the underlyings over long time periods. If the derivatives market is reasonably accurate, then both methods should come to similar conclusions. However, one has to admit that the derivatives market has been mistaken and is evolving. For instance, implied volatilities of (equity) index options showed only after the 1987 crash a systematic negative skew, however, the leverage effect has been similar in equity index dynamics before and after 1987. Therefore, let us finally spend a few words on the second method for checking whether the Radon-Nikodym derivatives of the putative risk neutral measures for the respective currency denominations are likely to be martingales or strict supermartingales. For this purpose one could employ a proxy of the GOP, e.g. a well diversified total return world index, see Platen and Rendek (2012a). In Platen and Rendek (2012b) the dynamics of such benchmarked saving accounts have been statistically analyzed and modelled. It turned out that a special case of our proposed model has been supported by that empirical analysis. Additionally, this study revealed some random market activity time. For long dated derivatives this randomness does not matter much because of the ergodicity of the market activity process. For short maturities, however, this extra source of uncertainty is important. It can be expected that such random market activity would increase in our model the curvature of the implied volatilities for short dated options. Forthcoming work on our model with random market activity time will 
demonstrate this effect in detail. It will also price and hedge long dated vanilla FX derivatives with lower prices than the risk neutral approach would suggest by exploiting the strict supermartingale property of the proposed model as it follows from the calibration of short dated options and long term historical observations.

\subsection{General Methodology}

We emphasize that it is not possible to confirm a model. According to Popper (2002) one can only falsify a model. Still we can gain more and more confidence in the ability of a model to reflect well the FX dynamics for our purposes, which is derivative pricing. We have so far developed a stochastic volatility model that is potentially able to fit the implied volatility surface of the FX market while maintaining a good level of analytical tractability. Moreover, our model is able to reflect well the observed systematic downward long term trends of benchmarked savings accounts as historically observed. It still works even in the absence of a risk neutral probability measure, whose existence depends on the inequality (10). The aim in this section is to calibrate the model to real options data and check on this basis if the market data support or reject the existence of the risk neutral probability measure and, thus, the risk neutral approach. In a Heston-like framework, the existence of the risk neutral measure is assumed by the choice of model for any value of the parameters of the model (see Remark 2). Thus, the calibration exercise in De Col et al. (2013) has no freedom to escape the classic risk neutral paradigm. What is of serious concern in De Col et al. (2013) is that one finds that the Feller condition (assuming the strict positivity of the volatility factors) is systematically violated by the calibrated parameters. This suggests that the Heston specification for a stochastic volatility model leads to ambiguous results when calibrated on real market data. Intuitively, the volatility spends too much time very close to zero under such condition. We also recall the paper of Drimus (2011) who calibrates both the Heston and the (single factor) $3 / 2$ model on $S \& P 500$ European options. In the $3 / 2$ model Drimus imposes some constraint on the parameters (most importantly a negative correlation in analogy with the Heston model) in order to enforce the existence of the risk neutral measure. The calibrated parameters reveal a degenerate dynamics $(\rho=-0.99)$ and indicate a severe problem in the specification of the model. Our model does not suffer from any such preimposed restrictions.

We will calibrate our model freely without imposing any condition on the correlation and we will let the data decide whether they lead us into the risk neutral world or into a more general modelling world. In this respect, the current paper seems to present the first stochastic volatility model that is calibrated on real market data without the (explicit or implicit) assumption that the risk neutral measure exists but is still adopting a coherent pricing methodology. We also note a particular feature of our model in terms of estimation: Even that we work under the historical or real world probability measure, we do not need to estimate the drift of the GOP, which would be an extremely difficult task since an extremely long dataset would be needed.

Using a real dataset of options from a major provider, we consider the implied volatility surfaces for USD/EUR, USD/JPY and EUR/JPY, as observed in the FX market on 22nd July 2010 and 2nd May $2013^{2}$, that is $N=3$ with $i=U S D, E U R, J P Y$. The volatility sample includes 4 expiry dates ranging from 1 month to 1 year for the datasets. The quotes follow the standard Delta quoting convention in the FX option market, we have quotes on DN (ATM), 25 Delta, and 10 Delta $^{3}$. We recall that our model shares the functional symmetry of the one in Heath and Platen

\footnotetext{
${ }^{2}$ We chose trading days close to the ones considered in De Col et al. (2013). As in their sample, the implied volatility surfaces as of 2 May 2013 are inverted with respect to the ones of 22 July 2010.

${ }^{3}$ In the FX market, implied volatilities surfaces are expressed in terms of maturity and Delta (see e.g. Wystup (2006), Clark (2011), De Col et al. (2013)).
} 
(2006), and it is, therefore, potentially able to fit simultaneously all the implied volatility surfaces of the triangle USD/EUR, USD/JPY and EUR/JPY in analogy with De Col et al. (2013).

The aim of this section is to investigate the inequality (10) for the three currencies under consideration, USD, EUR, and JPY. We calibrate the two-factor version of our model (i.e. $d=2$ ) to different books on 22nd July 2010 including two different panels corresponding to the whole dataset (including four maturities) and just one maturity. Since we deal with a time-homogeneous diffusion, the martingale property should not depend on the maturity: This is in line with the theoretical results of Bayraktar et al. (2012), who found that if the benchmarked saving accounts $\hat{B}^{i}$ is going to loose its martingale property eventually, it must loose its martingale property immediately.

\subsection{Calibration Results}

In Table 1, we present the calibrated parameters for the dataset on 22nd July 2010 including all the four maturities (1 month, 3 months, 6 months, 1 year) and display the quantity

$$
p_{k}^{i}=\frac{\sigma_{k}}{2}+\frac{\kappa_{k}}{\sigma_{k}}+\rho_{k} a_{k}^{i}, i \in\{U S D, E U R, J P Y\}, k=1,2 .
$$

For example, $p_{k}^{\text {For }}$ (resp. $p_{k}^{\text {Dom }}$ ) indicates the term $\sigma_{k} / 2+\kappa_{k} / \sigma_{k}+\rho_{k} a_{k}^{\text {For }}$ (resp. $\sigma_{k} / 2+\kappa_{k} / \sigma_{k}+$ $\rho_{k} a_{k}^{D o m}$ ) in (32) for the foreign (resp. domestic) currency. The corresponding benchmarked savings account is a true martingale if and only if these terms are non negative for all $k=1,2$. We note that for almost all currencies the quantity $p^{i}$ is negative for at least some $k$. This shows that our calibration leads beyond the risk neutral world, which is an extremely important finding. The risk neutral measure may potentially exist for some currencies, so that the risk neutral pricing formula may still be applicable for these currencies and short dated derivatives. However, in view of historically observed benchmarked savings accounts, which seem to form strict supermartingales, the risk neutral price may be not the minimal possible price for longer dated derivatives.

In Figures 1, 2 and 3 we plot the market implied volatilities against the model implied volatilities. Market implied volatilities are denoted by crosses, model implied volatilities by circles. The model yields overall a quite satisfactory fit what concerns the shape of the market implied volatility curves. Table 2 shows the numerical differences between market implied volatilities and model implied volatilities. The numerical error is in line with the usual performance of an advanced stochastic volatility model as in De Col et al. (2013) and it is plotted in Figures 4, 5 and 6.

In Table 3, we present the calibrated parameters for the dataset on 22nd July 2010 including only one maturity, where we consider separately the cases resp. 6 months and 1 year. We obtain qualitatively the same results as in the previous case, that is for almost all currencies the quantity $p^{i}$ is negative for at least some $k$. This goes in the direction of the theoretical results of Mijatović and Urusov (2012) and Bayraktar et al. (2012), who found that the strict local martingale property does not depend on the maturity.

To gain further confidence in the proposed model, we repeated the calibration experiment for a different dataset observed on 2nd May 2013, where the implied volatility surfaces are inverted with respect to the previous trading day. In Table 4, we show the results of the calibration for the 2-factor version of the model for the dataset that includes the following four maturities: 1 month, 3 months, 6 months, 1 year. Table 5 shows the numerical differences between market implied volatilities and model implied volatilities. In Figures 7, 8 and 9 we plot the market implied volatilities against the model implied volatilities, while the numerical errors are plotted in Figures 10, 11 and 12. We observe a failure of risk neutral pricing for most currencies. 
Summarizing the previous calibration results for options on exchange rates, we find that for most currencies the respective benchmarked savings accounts turn out to be strict supermartingales. This highlights the importance of being able to model and price claims under the real world probability measure and not having to rely on an assumed risk neutral probability measure.

\section{Conclusion and Future Research}

In this paper we have introduced a novel stochastic volatility model that is able to replicate stylized facts in vanilla FX options and allows one also to hedge payoffs in foreign currencies for a lower price than possible under classical risk neutral pricing. This has been achieved by using a multivariate version of the $3 / 2$ model that can generate strict local martingales where one has classically martingales. When the model has been calibrated for standard market days, we found that the risk neutral approach is not feasible for typical traded maturities, which constitutes a strong warning for the long standing practice to adopt the risk neutral pricing paradigm. Our contribution opens the door to further research, which is obvioulsy necessary in order to get a more detailed and global picture for the likely unsuitability of the risk neutral paradigm in other situations, in particular for derivatives with longer maturities.

\section{References}

Abramowitz, M. and Stegun, I. A. (1970). Handbook of Mathematical Functions. Dover Publications, New York, first edition.

Ahn, D. and Gao, B. (1999). A parametric nonlinear model of term structure dynamics. Review of Financial Studies, 12:721-762.

Andersen, L. B. G. and Piterbarg, V. V. (2007). Moment explosions in stochastic volatility models. Finance and Stochastics, 11:29-50.

Bakshi, G., Carr, P., and Wu, L. (2008). Stochastic risk premiums, stochastic skewness in currency options, and stochastic discount factors in international economies. Journal od Financial Economics, 87:132-156.

Bakshi, G., Ju, N., and Ou-Yang, H. (2006). Estimation of continuous-time models with an application to equity volatility dynamics. Journal of Financial Economics, 82:227-249.

Bayraktar, E., Kardaras, C., and Xing, H. (2012). Valuation equations for stochastic volatility models. SIAM Journal of Financial Mathematics, 3:351-373.

Beneder, R. and Elkenbracht-Huizing, M. (2003). Foreign exchange options and the volatility smile. Medium Econometrische Toepassingen, 2:30-36.

Carr, P. and Sun, J. (2007). A new approach for option pricing under stochastic volatility. Review of Derivatives Research, 10:87-150.

Castagna, A. (2010). FX Options and Smile Risk. Wiley.

Chan, L. and Platen, E. (2011). Exact Pricing and Hedging Formulas of Long Dated Variance Swaps under a 3/2 Volatility Model. QFRC-Research Paper, University of Technology Sydney.

Christensen, M. and Larsen, K. (2007). No arbitrage and the growth optimal portfolio. Stochastic Analysis and Applications, 25(1):255-280. 
Clark, I. (2011). Foreign Exchange Option Pricing: A Practitioner's Guide. Wiley.

Da Fonseca, J. and Grasselli, M. (2011). Riding on the smiles. Quantitative Finance, 11:1609-1632.

Da Fonseca, J., Grasselli, M., and Tebaldi, C. (2007). Option pricing when correlations are stochastic: an analytical framework. Review of Derivatives Research, 10(2):151-180.

De Col, A., Gnoatto, A., and Grasselli, M. (2013). Smiles all around: FX joint calibration in a multi-Heston model. Journal of Banking and Finance, 10:3799-3818.

Delbaen, F. and Schachermayer, W. (1995). The no-arbitrage property under a change of numeraire. Stochastics and Stochastic Reports, 53:213-226.

Drimus, G. G. (2011). Options on realized variance by transform methods: a non-affine stochastic volatility model. Quantitative Finance, 12:1-16.

Duffie, D., Filipovic, D., and Schachermayer, W. (2003). Affine processes and applications in finance. Annals of Applied Probability, 13:984-1053.

Feller, W. (1951). Two singular diffusion problems. Annals of Mathematics, 54:173-182.

Heath, D. and Platen, E. (2006). Currency derivatives under a minimal market model. The ICFAI Journal of Derivatives Marktes, 3:68-86.

Heston, S. (1997). A simple new formula for options with stochastic volatility. Technical report, Washington University of St. Louis.

Heston, S., Loewenstein, M., and Willard, G. A. (2007). Options and Bubbles. Review of Financial Studies, 20(2):359-390.

Heston, S. L. (1993). A closed-form solution for options with stochastic volatility with applications to bond and currency options. Review of Financial Studies, 6:327-343.

Hulley, H. (2010). The economic plausibility of strict local martingales in financial modelling. in Chiarella, C., Novikov, A. (eds), Contemporary Quantitative Finance, Springer, pages 53-75.

Itkin, A. and Carr, P. (2009). Pricing swaps and options on quadratic variation under stochastic time change models - discrete observations case. Review of Derivatives Research, forthcoming.

Jarrow, R. and Protter, P. (2010). Foreign Currency Bubbles. Preprint, Cornell University.

Jarrow, R., Protter, P., and Shimbo, K. (2010). Asset price bubbles in incomplete markets. Mathematical Finance, 20:145-156.

Karatzas, I. and Kardaras, C. (2007). The numéraire portfolio in semimartingale financial models. Finance and Stochastics, 4(11):447-493.

Karatzas, I. and Shreve, S. E. (1991). Brownian Motion and Stochastic Calculus. Springer-Verlag.

Lewis, A. L. (2000). Option Valuation Under Stochastic Volatility. Finance Press, Newport Beach, first edition.

Loewenstein, M. and Willard, G. A. (2000). Local martingales, arbitrage, and viability: Free snacks and cheap thrills. Economic Theory, 2(16):135-161.

Mijatović, A. and Urusov, M. (2012). On the martingale property of certain local martingales. Probability Theory and Related Fields, 152(1):1-30. 
Platen, E. (1997). A non-linear stochastic volatility model. Financial Mathematics Research Report No. FMRR 005-97, Center for Financial Mathematics, Australian National University, Canberra.

Platen, E. (2001). A minimal financial market model. In Trends in Mathematics, Birkhäuser, pages 293-301.

Platen, E. and Heath, D. (2010). A Benchmark Approach to Quantitative Finance. Springer-Verlag.

Platen, E. and Rendek, R. (2012a). Approximating the numéraire portfolio via naive diversification. Journal of Asset Management, 13, pages 34-50.

Platen, E. and Rendek, R. (2012b). The affine nature of aggregate wealth dynamics. QFRCResearch Paper No. 322, University of Technology Sydney.

Popper, K. R. (2002). The Logic of Scientific Discovery. Routledge. Originally published Logik der Forschung, Vienna (1935).

Protter, P. (2012). A Mathematical Theory of Financial Bubbles. Preprint, Columbia University.

Spencer, P. (2003). Coupon bond valuation with a non-affine discount yield model. Working Paper, Department of Economics, University of York.

Wystup, U. (2006). FX Options and Structured Products. Wiley.

\section{Appendix A: Proofs}

Proof of Theorem 1. Let us first recall that from (8), it follows that $V_{k}$ does not explode under the real world probability measure $\mathbb{P}$. We now check if $V_{k}$ explodes under the candidate risk neutral measure with strictly positive putative risk neutral probability: If this is the case, then the real world probability measure would not be equivalent to the putative risk neutral measure by Theorem 2.1 in Mijatović and Urusov (2012) (see also Section 3 in Bayraktar et al. (2012)). To check this we study the process

$$
d \tilde{V}_{k}(t)=\kappa_{k} \tilde{V}_{k}(t)\left(\theta_{k}-\tilde{V}_{k}(t)\right) d t-a_{k}^{i} \rho_{k} \tilde{V}_{k}^{2}(t) \sigma_{k} d t+\sigma_{k} \tilde{V}_{k}^{\frac{3}{2}}(t) d \tilde{W}_{k}(t)
$$

where

$$
d \tilde{W}_{k}(t)=d W_{k}(t)+a_{k}^{i} \rho_{k}\left(\tilde{V}_{k}(t)\right)^{1 / 2} d t
$$

is the stochastic differential of the process $\tilde{W}_{k}$, which should be a Wiener process under the putative risk neutral measure. Using the Feller explosion test as in (8) to the process $\tilde{V}_{k}(t)$ we find that for $-a_{k}^{i} \rho_{k}>\frac{\sigma_{k}}{2}+\frac{\kappa_{k}}{\sigma_{k}}$ the process $\tilde{V}_{k}(t)$ can explode, hence there is no equivalent risk neutral probability measure, which proves the first part of the theorem. The second part easily follows from the remark in Mijatović and Urusov (2012) following Theorem 2.1.

Proof of Proposition 1. For fixed $y^{1}$ one has

$$
\int_{\mathbb{R}} e^{\mathrm{i} \lambda^{0} y^{0}} \min \left(e^{-y^{0}}, K e^{-y^{1}}\right) d y^{0}=\int_{-\infty}^{y^{1}-\ln K} K e^{y^{0} \dot{i} \lambda^{0}-y^{1}} d y^{0}+\int_{y^{1}-\ln K}^{\infty} e^{y^{0}\left(\dot{i} \lambda^{0}-1\right)} d y^{0} .
$$

Now, it follows

$$
\int_{-\infty}^{y^{1}-\ln K} e^{i \lambda^{0} y^{0}} K e^{-y^{1}} d y^{0}=\frac{K^{1-i \lambda^{0}} e^{y^{1}\left(i \lambda^{0}-1\right)}}{i \lambda^{0}}
$$


subject to $\operatorname{Im}\left(\lambda^{0}\right)<0$. Also, we find

$$
\int_{y^{1}-\ln K}^{\infty} e^{y^{0}\left(\mathrm{i} \lambda^{0}-1\right)} d y^{0}=-\frac{K^{1-\mathfrak{i} \lambda^{0}} e^{y^{1}\left(\mathrm{i} \lambda^{0}-1\right)}}{\mathrm{i} \lambda^{0}-1},
$$

subject to $\operatorname{Im}\left(\lambda^{0}\right)>-1$. Hence

$$
\int_{\mathbb{R}} e^{\mathrm{i} \lambda^{0} y^{0}} \min \left(e^{-y^{0}}, K e^{-y^{1}}\right) d y^{0}=-\frac{K^{1-\mathrm{i} \lambda^{0}} e^{y^{1}\left(\mathrm{i} \lambda^{0}-1\right)}}{\mathrm{i} \lambda^{0}\left(\mathrm{i} \lambda^{0}-1\right)},
$$

subject to $-1<\operatorname{Im}\left(\lambda_{0}\right)<0$. We are now ready to compute the function $\hat{F}\left(\lambda^{0}, \lambda^{1}\right)$ :

$$
\begin{aligned}
\hat{F}\left(\lambda^{0}, \lambda^{1}\right) & =\int_{\mathbb{R}^{2}} e^{\mathrm{i} \lambda^{0} y^{0}+\mathrm{i} \lambda^{1} y^{1}} \min \left(e^{-y^{0}}, K e^{-y^{1}}\right) d y^{0} d y^{1} \\
& =-\frac{K^{1-\mathrm{i} \lambda^{0}}}{\mathrm{i} \lambda^{0}\left(\mathrm{i} \lambda^{0}-1\right)} \int_{\mathbb{R}} e^{\mathrm{i} \lambda^{1} y^{1}} e^{y^{1}\left(\mathrm{i} \lambda^{0}-1\right)} d y^{1} \\
& =-\frac{K^{1-\mathrm{i} \lambda^{0}}}{\mathrm{i} \lambda^{0}\left(\mathrm{i} \lambda^{0}-1\right)} \int_{\mathbb{R}} e^{y^{1}\left(\mathrm{i} \operatorname{Re}\left(\lambda^{0}\right)+\mathrm{i} \operatorname{Re}\left(\lambda^{1}\right)\right)} e^{\left(-\operatorname{Im}\left(\lambda^{0}\right)-\operatorname{Im}\left(\lambda^{1}\right)-1\right) y^{1}} d y^{1},
\end{aligned}
$$

so that we require $\operatorname{Im}\left(\lambda^{0}\right)+\operatorname{Im}\left(\lambda^{1}\right)=-1$. Since

$$
\int_{\mathbb{R}} e^{y^{1} \mathrm{i}\left(\operatorname{Re}\left(\lambda^{0}\right)+\operatorname{Re}\left(\lambda^{1}\right)\right)} d y^{1}=2 \pi \delta_{0}\left(\operatorname{Re}\left(\lambda^{0}\right)+\operatorname{Re}\left(\lambda^{1}\right)\right),
$$

the proof is complete.

Proof of Proposition 2. By the Feynman-Kac formula, $f$ satisfies the following PDE:

$$
\begin{aligned}
-\frac{\partial f}{\partial t}= & \sum_{j=0}^{1}\left(r^{j}+\frac{1}{2}\left(\mathbf{a}^{j}\right)^{\top} \operatorname{Diag}(\mathbf{V}) \mathbf{a}_{j}\right) \frac{\partial f}{\partial y^{j}} \\
& +\sum_{k=1}^{d} \kappa_{k} V_{k}\left(\theta_{k}-V_{k}\right) \frac{\partial f}{\partial V_{k}}+\frac{1}{2} \sum_{k=1}^{d} \sigma_{k}^{2} V_{k}^{3} \frac{\partial^{2} f}{\partial V_{k}^{2}}+\sum_{j=0}^{1} \sum_{k=1}^{d} a_{k}^{j} \sigma_{k} \rho_{k} V_{k}^{2} \frac{\partial^{2} f}{\partial y^{j} \partial V_{k}} \\
& +\sum_{j, l=0}^{1} \frac{1}{2}\left(\mathbf{a}^{j}\right)^{\top} \operatorname{Diag}(\mathbf{V}) \mathbf{a}^{l} \frac{\partial^{2} f}{\partial y^{j} \partial y^{l}},
\end{aligned}
$$

with boundary condition

$$
f\left(T, \lambda^{0}, \lambda^{1}, Y^{0}(T), Y^{1}(T), \mathbf{V}\right)=e^{\mathrm{i} \lambda^{0} Y^{0}(T)+\mathrm{i} \lambda^{1} Y^{1}(T)} .
$$

We guess the following separable form for the solution:

$$
f\left(t, \lambda^{0}, \lambda^{1}, y^{0}, y^{1}, \mathbf{V}\right)=e^{i \lambda^{0} y^{0}+\mathrm{i} \lambda^{1} y^{1}+\mathrm{i}(T-t)\left(r^{0} \lambda^{0}+r^{1} \lambda^{1}\right)} L(t, \boldsymbol{\lambda}, \mathbf{V}),
$$

so that $L$ satisfies the following PDE

$$
\begin{aligned}
-\frac{\partial L}{\partial t}= & \left(\sum_{j=0}^{1} \frac{1}{2}\left(\mathbf{a}^{j}\right)^{\top} \operatorname{Diag}(\mathbf{V}) \mathbf{a}^{j}\left(i \lambda^{j}\right)-\sum_{j, l=0}^{1} \frac{1}{2}\left(\mathbf{a}^{j}\right)^{\top} \operatorname{Diag}(\mathbf{V}) \mathbf{a}^{l} \lambda^{j} \lambda^{l}\right) L \\
& +\sum_{k=1}^{d} \kappa_{k} V_{k}\left(\theta_{k}-V_{k}\right) \frac{\partial L}{\partial V_{k}}+\frac{1}{2} \sum_{k=1}^{d} \sigma_{k}^{2} V_{k}^{3} \frac{\partial^{2} L}{\partial V_{k}^{2}} \\
& +\sum_{j=0}^{1} \sum_{k=1}^{d} a_{k}^{j} \sigma_{k} \rho_{k} V_{k}^{2} \frac{\partial L}{\partial V_{k}}\left(i \lambda^{j}\right),
\end{aligned}
$$


with boundary condition

$$
L(T, \boldsymbol{\lambda}, \mathbf{V})=1 \text {. }
$$

Now we note that $\left(\mathbf{a}^{j}\right)^{\top} \operatorname{Diag}(\mathbf{V}) \mathbf{a}^{l}=\sum_{k=1}^{d} a_{k}^{j} V_{k} a_{k}^{j}$, and hence

$$
\sum_{j, l=0}^{1}\left(\mathbf{a}^{j}\right)^{\top} \operatorname{Diag}(\mathbf{V}) \mathbf{a}^{l} \lambda^{j} \lambda^{l}=\sum_{k=1}^{d} V_{k}\left\langle\mathbf{a}_{\mathbf{k}}, \boldsymbol{\lambda}\right\rangle^{2}
$$

where for $k=1, . ., d, \mathbf{a}_{\mathbf{k}}=\left(a_{k}^{0}, a_{k}^{1}\right)^{\top} \in \mathbb{R}^{2}$ and $\left\langle\mathbf{a}_{\mathbf{k}}, \boldsymbol{\lambda}\right\rangle^{2}=\sum_{j, l=0}^{1} a_{k}^{j} \lambda^{j} a_{k}^{l} \lambda^{l}$.

Also, we have

$$
\begin{aligned}
\sum_{j=0}^{1}\left(\mathbf{a}^{j}\right)^{\top} \operatorname{Diag}(\mathbf{V}) \mathbf{a}^{j} \lambda^{j} & =\sum_{k=1}^{d} \sum_{j=0}^{1} V_{k} a_{k}^{j} a_{k}^{j} \lambda^{j} \\
& =\sum_{k=1}^{d} V_{k} \sum_{j=0}^{1}\left(a_{k}^{j}\right)^{2} \lambda^{j}
\end{aligned}
$$

and hence

$$
\begin{aligned}
-\frac{\partial L}{\partial t}= & \sum_{k=1}^{d}\left(\frac{1}{2} \sigma_{k}^{2} V_{k}^{3} \frac{\partial^{2} L}{\partial V_{k}^{2}}+\left(\kappa_{k} V_{k}\left(\theta_{k}-V_{k}\right)+i \rho_{k} \sigma_{k}\left\langle\boldsymbol{\lambda}, \mathbf{a}_{\mathbf{k}}\right\rangle V_{k}^{2}\right) \frac{\partial L}{\partial V_{k}}\right. \\
& \left.+L V_{k}\left(\frac{i}{2} \sum_{j=0}^{1}\left(a_{k}^{j}\right)^{2} \lambda^{j}-\frac{1}{2}\left\langle\mathbf{a}_{\mathbf{k}}, \boldsymbol{\lambda}\right\rangle^{2}\right)\right) .
\end{aligned}
$$

Now we set $\tau=T-t, c_{k}=-\left(\frac{i}{2} \sum_{j=0}^{1}\left(a_{k}^{j}\right)^{2} \lambda^{j}-\frac{1}{2}\left\langle\mathbf{a}_{\mathbf{k}}, \lambda\right\rangle^{2}\right)$ and we guess that the solution of the $\mathrm{PDE}$ is of the form

$$
L=\prod_{k=1}^{d} L_{k}
$$

where

$$
\frac{\partial L_{k}}{\partial \tau}=\left(\frac{1}{2} \sigma_{k}^{2} V_{k}^{3} \frac{\partial^{2} L_{k}}{\partial V_{k}^{2}}+\left(\kappa_{k} V_{k}\left(\theta_{k}-V_{k}\right)+i \rho_{k} \sigma_{k}\left\langle\boldsymbol{\lambda}, \mathbf{a}_{\mathbf{k}}\right\rangle V_{k}^{2}\right) \frac{\partial L_{k}}{\partial V_{k}}-L_{k} V_{k} c_{k}\right)
$$

Set $s_{k}=\frac{\tau \sigma_{k}^{2}}{2}$, and $L_{k}\left(V_{k}, \tau\right)=f_{k}\left(V_{k}, s_{k}\right)$, then

$$
\frac{\partial f_{k}}{\partial s_{k}}=V_{k}^{3} \frac{\partial^{2} f_{k}}{\partial V_{k}^{2}}+\left(-\hat{\theta}_{k} V_{k}^{2}+\tilde{\omega}_{k} V_{k}\right) \frac{\partial f_{k}}{\partial V_{k}}-f_{k} V_{k} \tilde{c}_{k}
$$

where

$$
\begin{aligned}
\tilde{\omega}_{k} & =2 \frac{\kappa_{k} \theta_{k}}{\sigma_{k}^{2}} \\
\hat{\theta}_{k} & =\frac{2 \kappa_{k}}{\sigma_{k}^{2}}-\frac{2 \mathbf{i} \rho_{k}\left\langle\lambda, \mathbf{a}_{\mathbf{k}}\right\rangle}{\sigma_{k}} \\
\tilde{c}_{k} & =\frac{2 c_{k}}{\sigma_{k}^{2}} .
\end{aligned}
$$


We now employ similar changes of variables as in Lewis (2000) p. 332, that is

$$
\begin{aligned}
y_{k} & =\frac{\tilde{w}_{k}}{V_{k}} \\
t_{k} & =\tilde{\omega}_{k} s_{k},
\end{aligned}
$$

and set $u_{k}\left(y_{k}, t_{k}\right)=f_{k}\left(V_{k}, s_{k}\right)$, to get

$$
\frac{\partial u_{k}}{\partial t_{k}}=y_{k} \frac{\partial^{2} u_{k}}{\partial y_{k}^{2}}+\left(2+\hat{\theta}_{k}-y_{k}\right) \frac{\partial u_{k}}{\partial y_{k}}-\frac{\tilde{c}_{k}}{y_{k}} u_{k}
$$

subject to $u_{k}\left(y_{k}, 0\right)=1$. Next, we let $u_{k}\left(y_{k}, t_{k}\right)=y_{k}^{\nu_{k}} \exp \left(\left(1-\nu_{k}\right) t_{k}\right) g_{k}\left(y_{k}, t_{k}\right)$, choosing $\nu_{k}$ to satisfy the equation

$$
\nu_{k}^{2}+\left(1+\hat{\theta}_{k}\right) \nu_{k}-\tilde{c}_{k}=0
$$

We take

$$
\nu_{k}=-\mu_{k}-\delta_{k},
$$

where

$$
\mu_{k}=\frac{1}{2}\left(1+\hat{\theta}_{k}\right), \delta_{k}=\sqrt{\mu_{k}^{2}+\tilde{c}_{k}},
$$

and $g_{k}$ satisfies the equation

$$
\frac{\partial g_{k}}{t_{k}}=\left(y_{k} g_{k}\right)_{y_{k} y_{k}}-\left(\left(y_{k}+\beta_{k}\right) g_{k}\right)_{y_{k}}
$$

subject to $g_{k}\left(y_{k}, 0\right)=y_{k}^{-\nu_{k}}$, where $\beta_{k}=1+2 \delta_{k}$. Equation (37) can be solved as in Lemma 1 in Feller (1951), to obtain

$$
G_{k}\left(x_{k}, t_{k}\right)=\Gamma\left(1-\nu_{k}\right)\left(\frac{x_{k} e^{t_{k}}}{1+x_{k}\left(e^{t_{k}}-1\right)}\right)^{\nu_{k}-1}\left(1+x_{k}\left(e^{t_{k}}-1\right)\right)^{-\beta_{k}}
$$

where

$$
G_{k}\left(x_{k}, t_{k}\right)=\int_{0}^{\infty} e^{-x_{k} y} g_{k}\left(y, t_{k}\right) d y .
$$

This Laplace transform is easily inverted to obtain the following result for $u_{k}$, which matches with the univariate result from Lewis (2000), p. 54 (see also , Carr and Sun (2007), Theorem 3.1):

$$
u_{k}\left(y_{k}, t_{k}\right)=\frac{\Gamma\left(\beta_{k}-\alpha_{k}\right)}{\Gamma\left(\beta_{k}\right)}\left(\frac{y_{k}}{e^{t_{k}}-1}\right)^{\alpha_{k}} M\left(\alpha_{k}, \beta_{k},-\frac{y_{k}}{e^{t_{k}}-1}\right),
$$

which completes the first part of the proof.

Let us now turn our attention to the regularity of the characteristic function on $\mathcal{Z}$. Note that for pricing, we are interested in the function

$$
\begin{aligned}
\Psi_{t}(-\mathbf{i} \boldsymbol{\lambda}) & =\mathbb{E}_{t}\left(\exp \left(-i \lambda^{0} Y^{0}(T)-i \lambda^{1} Y^{1}(T)\right)\right) \\
& =f(t,-\boldsymbol{\lambda}, \mathbf{y}, \mathbf{V})
\end{aligned}
$$

Therefore, we have to replace $\boldsymbol{\lambda}$ by $-\boldsymbol{\lambda}$ in the formula (27) giving $c_{k}$. The discontinuity occurs along the imaginary axes, so

$$
\lambda^{j}=\mathrm{i} \operatorname{Im}\left(\lambda^{j}\right), \quad j=0,1 .
$$


We arrive at the following formula:

$$
\begin{aligned}
c_{k}= & -\frac{i}{2} \sum_{j=0}^{1}\left(a_{k}^{j}\right)^{2}\left(-\lambda^{j}\right)+\frac{1}{2} \sum_{j, l=0}^{1} a_{k}^{j} a_{k}^{l}\left(-\lambda^{j}\right)\left(-\lambda^{l}\right) \\
= & -\frac{i}{2} \sum_{j=0}^{1}\left(a_{k}^{j}\right)^{2}\left(-\mathbf{I} \operatorname{Im}\left(\lambda^{j}\right)\right)+\frac{1}{2} \sum_{j, l=0}^{1} a_{k}^{j} a_{k}^{l} \dot{\mathrm{i}}^{2} \operatorname{Im}\left(\lambda^{j}\right) \operatorname{Im}\left(\lambda^{l}\right) \\
= & -\frac{1}{2}\left(\sum_{j=0}^{1}\left(a_{k}^{j}\right)^{2} \operatorname{Im}\left(\lambda^{j}\right)+\sum_{j, l=0}^{1} a_{k}^{j} a_{k}^{l} \operatorname{Im}\left(\lambda^{j}\right) \operatorname{Im}\left(\lambda^{l}\right)\right) \\
= & -\frac{1}{2}\left(\left(a_{k}^{0}\right)^{2} \operatorname{Im}\left(\lambda^{0}\right)+\left(a_{k}^{1}\right)^{2} \operatorname{Im}\left(\lambda^{1}\right)\right. \\
& \left.+\left(a_{k}^{0}\right)^{2}\left(\operatorname{Im}\left(\lambda^{0}\right)\right)^{2}+2 a_{k}^{1} a_{k}^{0} \operatorname{Im}\left(\lambda^{0}\right) \operatorname{Im}\left(\lambda^{1}\right)+\left(a_{k}^{1}\right)^{2}\left(\operatorname{Im}\left(\lambda^{1}\right)\right)^{2}\right) .
\end{aligned}
$$

Substituting $\operatorname{Im}\left(\lambda^{0}\right)+\operatorname{Im}\left(\lambda^{1}\right)=-1$, which defines the admissible domain in (17), we get

$$
c_{k}=-\frac{1}{2} \operatorname{Im}\left(\lambda^{0}\right)\left(1+\operatorname{Im}\left(\lambda^{0}\right)\right)\left(a_{k}^{0}-a_{k}^{1}\right)^{2} .
$$

From (17) we have $\operatorname{Im}\left(\lambda^{0}\right)+1>0$, and hence $c_{k} \geq 0$. Then the argument in Lewis (2000) (see page 55) applies, i.e. $\theta^{k}$ is real along the imaginary axes, $\lambda^{j}=i \operatorname{Im}\left(\lambda^{j}\right), j=0,1$, i.e. $\delta_{k}$ is real and satisfies $\delta_{k} \geq\left|\mu_{k}\right|$. Consequently, $\alpha_{k} \geq 0$ and $\beta_{k} \geq 1$. Therefore, no discontinuities occur on $\mathcal{Z}$ since the boundary flux at the origin vanishes (see Lewis (2000) page 333).

\section{Appendix B: Images and Tables}



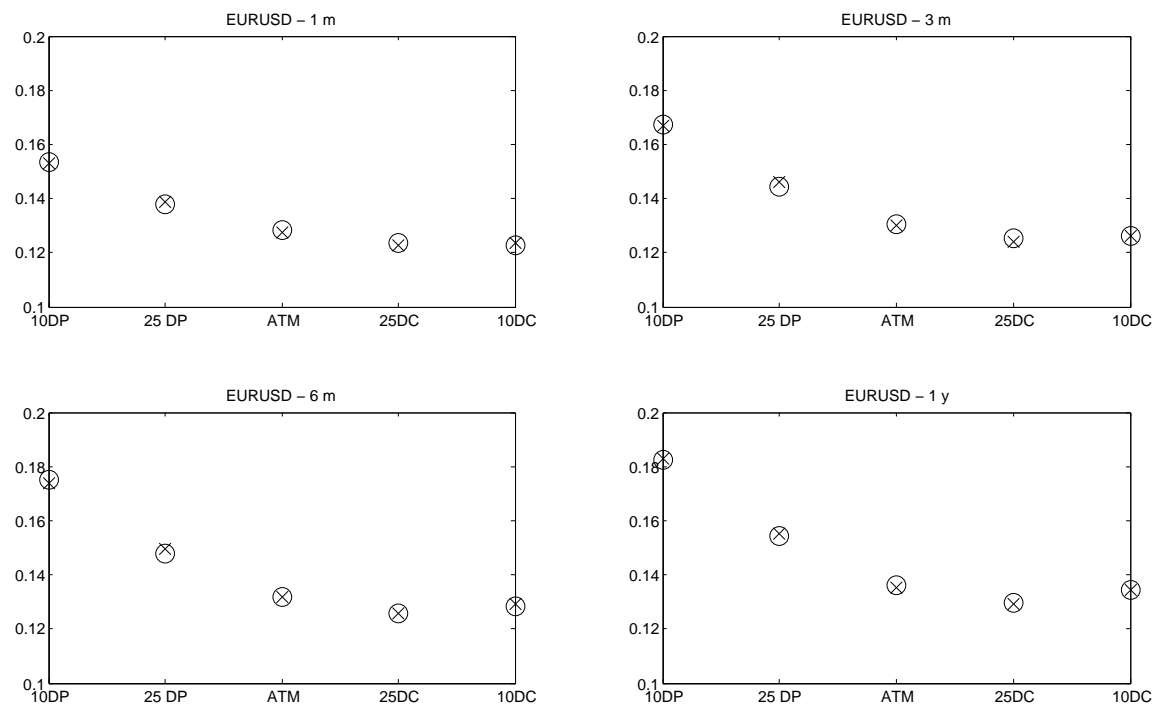

Figure 1: Calibration of the USD/EUR implied volatility surface. Market data as of 22nd July 2010. Market volatilities are denoted by crosses, model volatilities are denoted by circles. We include 4 maturities: 1 month, 3 months, 6 months, 1 year. Moneyness levels follow the standard Delta quoting convention in the FX option market. DC and DP stand for "Delta call" and "Delta put", respectively. Model parameters are given in Table 1.
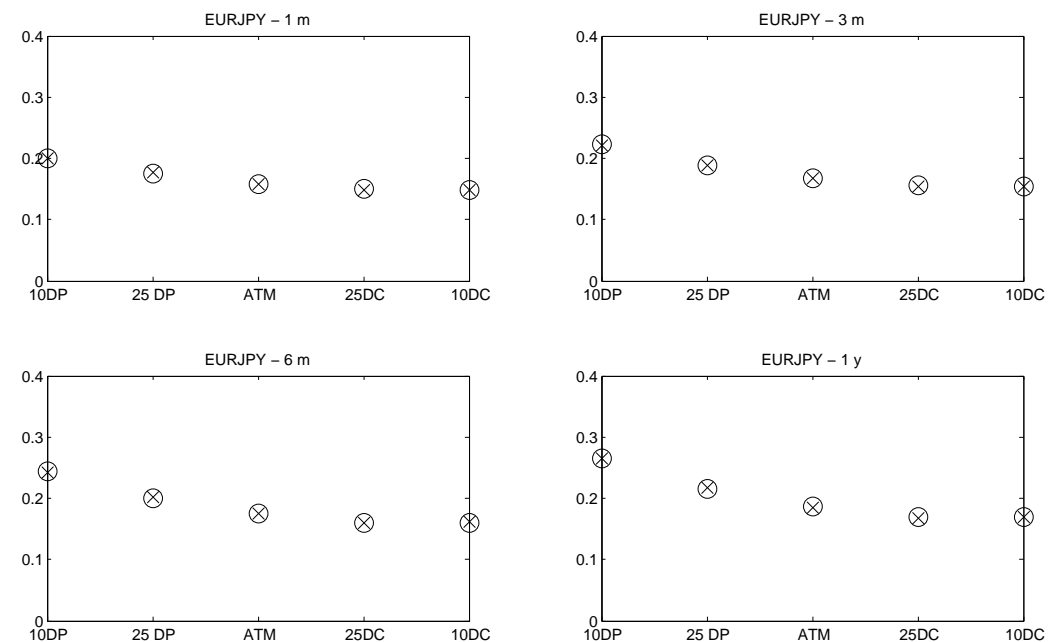

Figure 2: Calibration of the JPY/EUR implied volatility surface. Market data as of 22nd July 2010. Market volatilities are denoted by crosses, model volatilities are denoted by circles. We include 4 maturities: 1 month, 3 months, 6 months, 1 year. Moneyness levels follow the standard Delta quoting convention in the FX option market. DC and DP stand for "Delta call" and "Delta put", respectively. Model parameters are given in Table 1. 

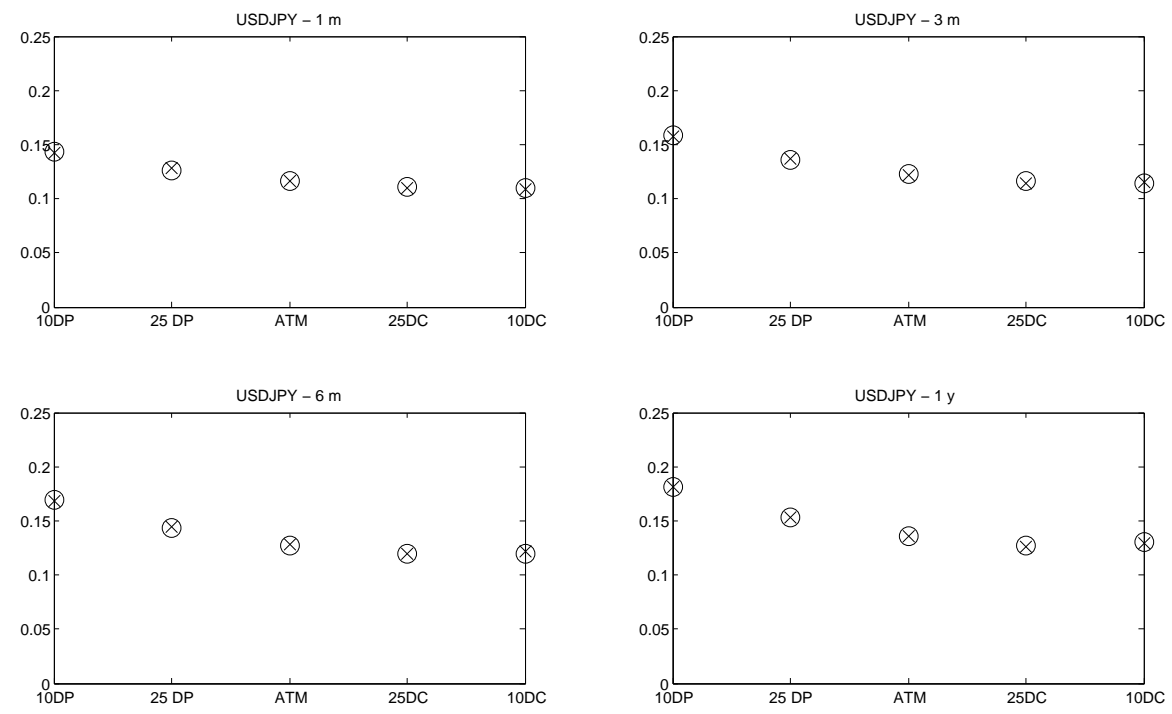

Figure 3: Calibration of the JPY/USD implied volatility surface. Market data as of 22nd July 2010. Market volatilities are denoted by crosses, model volatilities are denoted by circles. We include 4 maturities: 1 month, 3 months, 6 months, 1 year. Moneyness levels follow the standard Delta quoting convention in the FX option market. DC and DP stand for "Delta call" and "Delta put", respectively. Model parameters are given in Table 1.

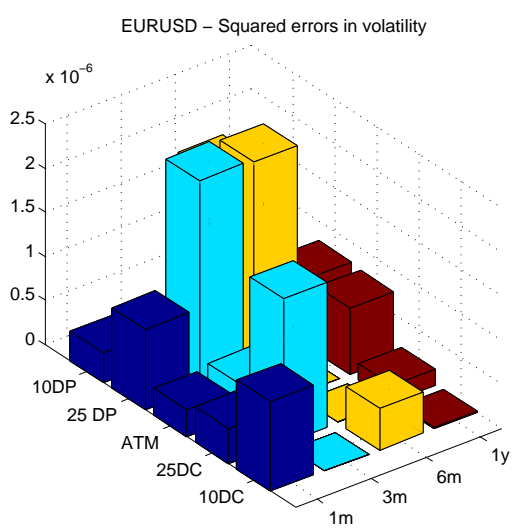

Figure 4: Squared error for the USD/EUR implied volatility surface. Market data as of 22nd July 2010. We include 4 maturities: 1 month, 3 months, 6 months, 1 year. Moneyness levels follow the standard Delta quoting convention in the FX option market. DC and DP stand for "Delta call" and "Delta put", respectively. Model parameters are given in Table 1. 


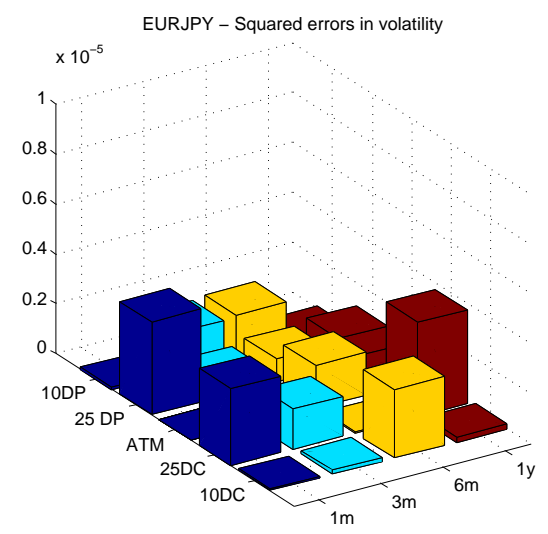

Figure 5: Squared error for the JPY/EUR implied volatility surface. Market data as of 22nd July 2010. We include 4 maturities: 1 month, 3 months, 6 months, 1 year. Moneyness levels follow the standard Delta quoting convention in the FX option market. DC and DP stand for "Delta call" and "Delta put", respectively. Model parameters are given in Table 1.

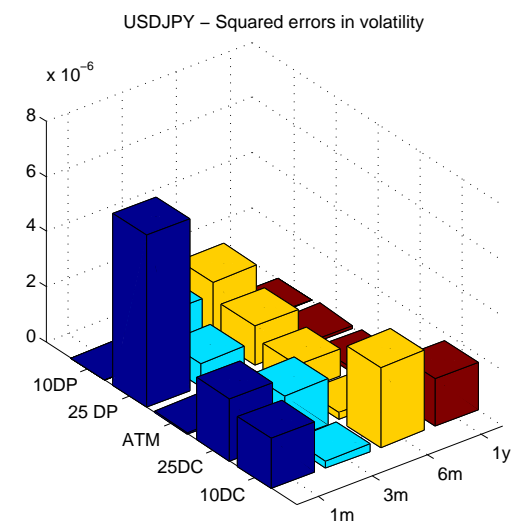

Figure 6: Squared error for the USD/JPY implied volatility surface. Market data as of 22nd July 2010. We include 4 maturities: 1 month, 3 months, 6 months, 1 year. Moneyness levels follow the standard Delta quoting convention in the FX option market. DC and DP stand for "Delta call" and "Delta put", respectively. Model parameters are given in Table 1. 

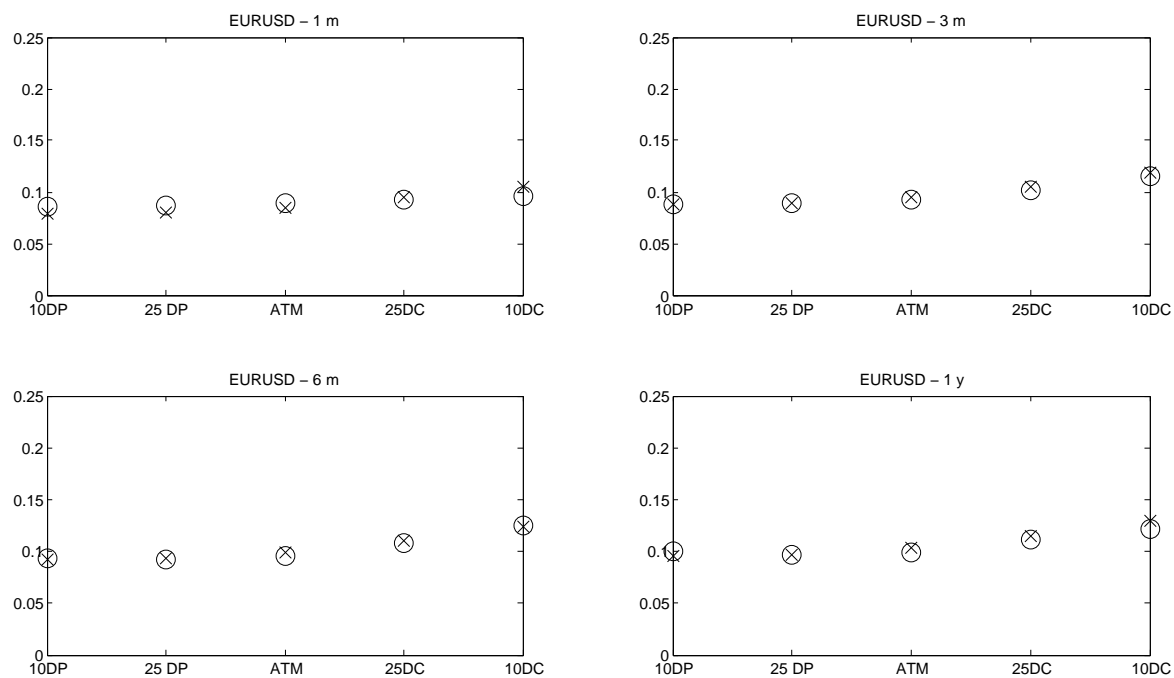

Figure 7: Calibration of the USD/EUR implied volatility surface. Market data as of 2nd May 2013. Market volatilities are denoted by crosses, model volatilities are denoted by circles. We include 4 maturities: 1 month, 3 months, 6 months, 1 year. Moneyness levels follow the standard Delta quoting convention in the FX option market. DC and DP stand for "Delta call" and "Delta put", respectively. Model parameters are given in Table 4. 

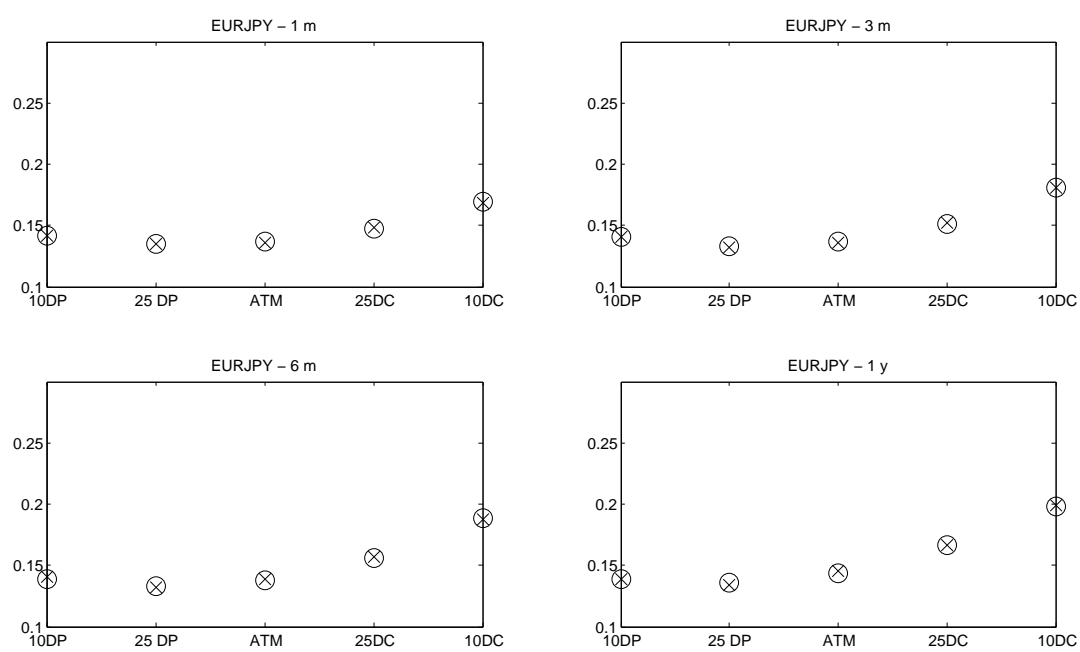

Figure 8: Calibration of the JPY/EUR implied volatility surface. Market data as of 2nd May 2013. Market volatilities are denoted by crosses, model volatilities are denoted by circles. We include 4 maturities: 1 month, 3 months, 6 months, 1 year. Moneyness levels follow the standard Delta quoting convention in the FX option market. DC and DP stand for "Delta call" and "Delta put", respectively. Model parameters are given in Table 4. 

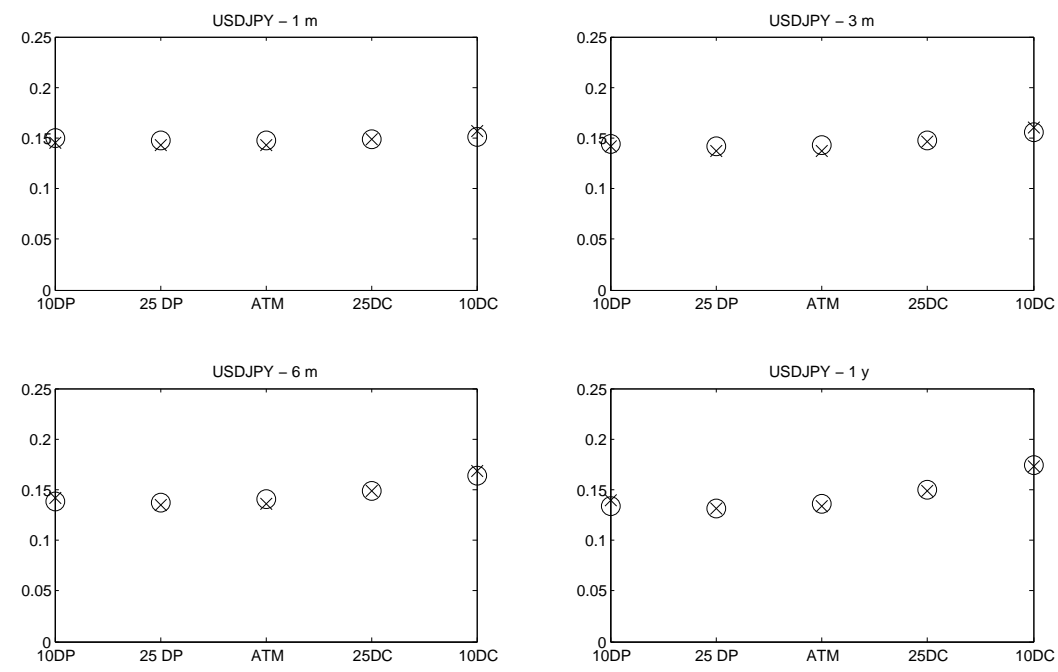

Figure 9: Calibration of the JPY/USD implied volatility surface. Market data as of 2nd May 2013. Market volatilities are denoted by crosses, model volatilities are denoted by circles. We include 4 maturities: 1 month, 3 months, 6 months, 1 year. Moneyness levels follow the standard Delta quoting convention in the FX option market. DC and DP stand for "Delta call" and "Delta put", respectively. Model parameters are given in Table 4.

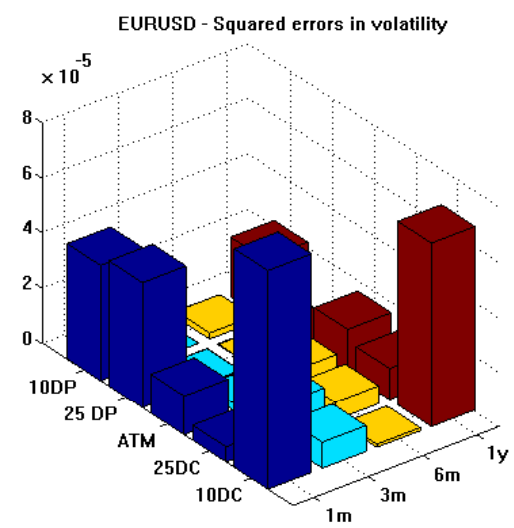

Figure 10: Squared error for the USD/EUR implied volatility surface. Market data as of 2nd May 2013. We include 4 maturities: 1 month, 3 months, 6 months, 1 year. Moneyness levels follow the standard Delta quoting convention in the FX option market. DC and DP stand for "Delta call" and "Delta put", respectively. Model parameters are given in Table 4. 


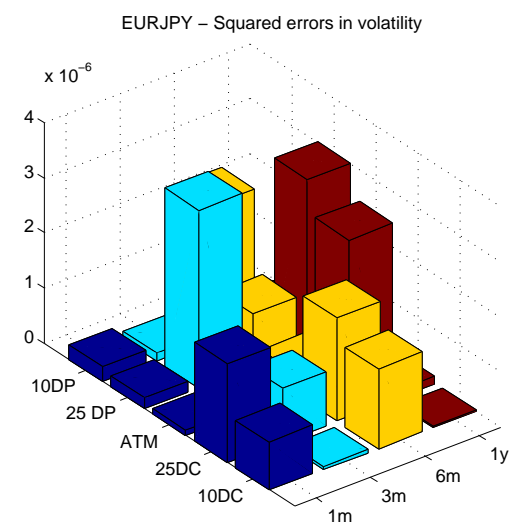

Figure 11: Squared error for the JPY/EUR implied volatility surface. Market data as of 2nd May 2013. We include 4 maturities: 1 month, 3 months, 6 months, 1 year. Moneyness levels follow the standard Delta quoting convention in the FX option market. DC and DP stand for "Delta call" and "Delta put", respectively. Model parameters are given in Table 4 .

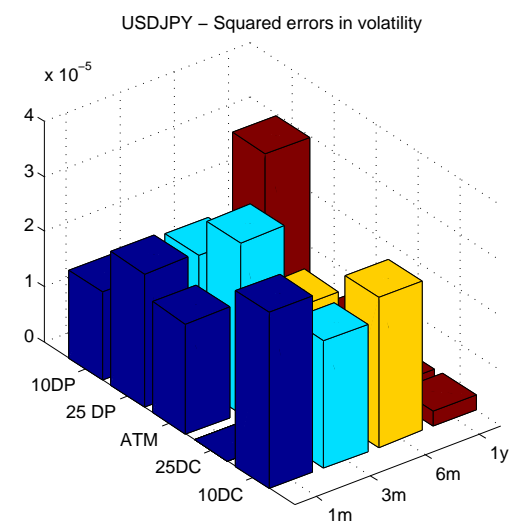

Figure 12: Squared error for the USD/JPY implied volatility surface. Market data as of 2nd May 2013. We include 4 maturities: 1 month, 3 months, 6 months, 1 year. Moneyness levels follow the standard Delta quoting convention in the FX option market. DC and DP stand for "Delta call" and "Delta put", respectively. Model parameters are given in Table 4. 


\begin{tabular}{cccc} 
Parameters & EURUSD & EURJPY & USDJPY \\
\hline$V_{1}$ & 0.771355 & 1.055712 & 1.636934 \\
$V_{2}$ & 1.093255 & 1.298630 & 1.202518 \\
$\kappa_{1}$ & -1.330424 & -1.663447 & -1.728106 \\
$\kappa_{2}$ & 0.792380 & 0.93503 & $-0,030107$ \\
$\sigma_{1}$ & 3.657986 & 3.62715 & 3.26299 \\
$\sigma_{2}$ & 1.395227 & 1.43802 & 1.158547 \\
$\theta_{1}$ & -2.260868 & -2.40059 & -2.193273 \\
$\theta_{2}$ & 0.98374 & 1.18666 & 0.110921 \\
$\rho_{1}$ & -0.36164 & -0.44632 & -0.465384 \\
$\rho_{2}$ & -0.740197 & -0.74939 & -0.394187 \\
$a_{1}^{\text {dom }}$ & 1.110667 & 1.253862 & 1.385031 \\
$a_{2}^{\text {dom }}$ & 1.756288 & 1.836335 & 1.473142 \\
$a_{1}^{\text {for }}$ & 0.975045 & 1.118780 & 1.325870 \\
$a_{2}^{\text {for }}$ & 1.735402 & 1.800107 & 1.406883 \\
Res. norm. & $1.39 \mathrm{e}-05$ & $2.39 \mathrm{e}-05$ & $2.48 \mathrm{e}-5$ \\
\hline$p_{1}^{\text {For }}$ & 1.112671 & 0.795337 & 0.48485 \\
$p_{2}^{\text {For }}$ & -0.01900 & -0.00691 & -0.001289 \\
$p_{1}^{\text {Dom }}$ & 1.063624 & 0.855627 & 0.457320 \\
$p_{2}^{\text {Dom }}$ & -0.034463 & 0.020238 & -0.027407 \\
\hline
\end{tabular}

Table 1: This table reports the results of the calibration of the model in the two factor case with market data as of 22nd July 2010. We include 4 maturities: 1 month, 3 months, 6 months, 1 year. For each column we show the calibrated parameters corresponding to a different pair of the triangle EUR/USD/JPY. We follow the convention FORDOM (e.g. EURUSD means that USD is the domestic currency, EUR is the foreign one). Strikes range in the set 10DP (i.e. 10 Delta Put), 25DP, ATM, 25DC, 10DC (for the market convention we refer to De Col et al. (2013)). The reference exchange rates are $S^{E U R U S D}(0)=1.2903, S^{E U R J P Y}(0)=112.29, S^{U S D J P Y}(0)=87.026$. Res. norm. is the residual of the objective function for the given set of parameters. $p_{k}^{F o r}$ (resp. $p_{k}^{D o m}$ ) indicates the term $\sigma_{k} / 2+\kappa_{k} / \sigma_{k}+\rho_{k} a_{k}^{F o r}$ (resp. $\sigma_{k} / 2+\kappa_{k} / \sigma_{k}+\rho_{k} a_{k}^{D o m}$ ) in (32) for the foreign (resp. domestic) currency. The corresponding benchmarked savings account is a true martingale if and only if these terms are non negative for all $k=1,2$. 
Vol Differences for EURUSD

\begin{tabular}{ccccc}
\hline & $1 \mathrm{~m}$ & $3 \mathrm{~m}$ & $6 \mathrm{~m}$ & $1 \mathrm{y}$ \\
\hline 10DP & -0.00057 & -0.00039 & -0.00137 & $4.61 \mathrm{e}-05$ \\
25DP & 0.00095 & 0.00153 & 0.00152 & 0.00088 \\
ATM & -0.00055 & -0.00059 & $5.54 \mathrm{e}-05$ & -0.00086 \\
25DC & -0.00064 & -0.001281 & $2.54 \mathrm{e}-05$ & -0.00042 \\
10DC & 0.001008 & $-3.52 \mathrm{e}-05$ & 0.00068 & 0.00011 \\
\hline \hline Vol Differences for EURJPY & & & & \\
& & & & \\
\hline & $1 \mathrm{~m}$ & $3 \mathrm{~m}$ & $6 \mathrm{~m}$ & $1 \mathrm{y}$ \\
\hline 10DP & -0.00027 & -0.00125 & -0.00129 & 0.00023 \\
25DP & 0.00192 & 0.00087 & 0.00096 & 0.00104 \\
ATM & $-4,53 \mathrm{e}-05$ & 0.00049 & 0.00130 & -0.00069 \\
25DC & -0.00174 & -0.00126 & -0.00013 & -0.00194 \\
10DC & -0.00024 & 0.00039 & 0.001655 & 0.00045 \\
\hline Vol Differences for USDJPY & & & & \\
& & & & \\
\hline 10DP & $1 \mathrm{~m}$ & $3 \mathrm{~m}$ & $6 \mathrm{~m}$ & $1 \mathrm{y}$ \\
25DP & $-8.02 \mathrm{e}-05$ & -0.00126 & -0.00142 & -0.00013 \\
ATM & 0.00249 & 0.00091 & 0.00119 & 0.00030 \\
25DC & -0.00027 & -0.00045 & 0.00099 & 0.00037 \\
10DC & -0.00150 & -0.00127 & 0.00051 & -0.00054 \\
\hline \hline
\end{tabular}

Table 2: This table reports the differences between market implied volatilities and model implied volatilities in the two factor case with market data as of 22nd July 2010 corresponding to a different pair of the triangle EUR/USD/JPY. We include 4 maturities: 1 month, 3 months, 6 months, 1 year. We follow the convention FORDOM (e.g. EURUSD means that USD is the domestic currency, EUR is the foreign one). Strikes range in the set 10DP (i.e. 10 Delta Put), 25DP, ATM, 25DC, 10DC (for the market convention we refer to De Col et al. (2013)). The reference exchange rates are $S^{E U R U S D}(0)=1.2903, S^{E U R J P Y}(0)=112.29, S^{U S D J P Y}(0)=87.026$. 


\begin{tabular}{ccccccc} 
Parameters & EURUSD 6m & EURUSD 1y & EURJPY 6m & EURJPY 1y & USDJPY 6m & USDJPY 1y \\
\hline$V_{1}$ & 1.694192 & 1.706194 & 1.715061 & 1.715432 & 1.77986 & 1.63301 \\
$V_{2}$ & 1.052543 & 0.678113 & 0.740801 & 0.657344 & 1.467089 & 1.271879 \\
$\kappa_{1}$ & 0.169056 & 0.223676 & 0.253533 & 0.253377 & -1.511631 & $-1 ; 73456$ \\
$\kappa_{2}$ & 0.394588 & 0.52102 & 0.309121 & 0.338675 & -0.19086 & 0.096019 \\
$\sigma_{1}$ & 3.126389 & 3.13396 & 3.15722 & 3.156955 & 4.045071 & 3.23359 \\
$\sigma_{2}$ & 1.554624 & 1.143817 & 1.168906 & 1.08465 & 1.527235 & 1.36443 \\
$\theta_{1}$ & -0.00399 & -0.00298 & 0.000646 & 0.00068 & -2.31880 & -2.18475 \\
$\theta_{2}$ & 0.050109 & 0.01489 & 0.044354 & 0.039707 & 0.104962 & 0.111806 \\
$\rho_{1}$ & -0.07963 & -0.01811 & -0.98946 & -0.991027 & -0.789816 & -0.63741 \\
$\rho_{2}$ & -0.96265 & -0.89118 & -0.94283 & -0.865760 & -0.412228 & -0.49477 \\
$a_{1}^{\text {dom }}$ & 1.477788 & 1.503462 & 1.379132 & 1.375549 & 0.91261 & 1.39159 \\
$a_{2}^{\text {dom }}$ & 1.435051 & 1.363557 & 1.492297 & 1.48240 & 1.541205 & 1.45855 \\
$a_{1}^{\text {for }}$ & 1.364904 & 1.332386 & 1.419632 & 1.423707 & 0.810048 & 1.338211 \\
$a_{2}^{\text {for }}$ & 1.376103 & 1.289862 & 1.31580 & 1.29736 & 1.458585 & 1.354850 \\
Res. norm. & $1.01 \mathrm{e}-9$ & $2.02 \mathrm{e}-5$ & $3,65 \mathrm{e}-06$ & $1,45 \mathrm{e}-8$ & $8.6 \mathrm{e}-8$ & $2,02 \mathrm{e}-5$ \\
\hline$p_{1}^{\text {For }}$ & 1.50856 & 1.614220 & 0.254246 & 0.247804 & 1.00904 & 0.22738 \\
$p_{2}^{\text {For }}$ & -0.29358 & -0.122083 & -0.391689 & -0.2686420 & 0.03737 & 0.08225 \\
$p_{1}^{\text {Dom }}$ & 1.49957 & 1.611122 & 0.29431 & 0.295530 & 0.928042 & 0.19335 \\
$p_{2}^{\text {Dom }}$ & $-0,35033$ & -0.18775 & -0.55809 & -0.428834 & 0.003314 & 0.03093 \\
\hline
\end{tabular}

Table 3: This table reports the results of the calibration of the model in the two factor case with market data as of 22nd July 2010. For each column we show the calibrated parameters corresponding to a different pair of the triangle EUR/USD/JPY by including only one maturity, where we consider separately resp. 6 months and 1 year. We follow the convention FORDOM (e.g. EURUSD means that USD is the domestic currency, EUR is the foreign one). Strikes range in the set 10DP (i.e. 10 Delta Put), 25DP, ATM, 25DC, 10DC (for the market convention we refer to De Col et al. (2013)). The reference exchange rates are $S^{E U R U S D}(0)=1.2903, S^{E U R J P Y}(0)=112.29$, $S^{U S D J P Y}(0)=87.026$. Res. norm. is the residual of the objective function for the given set of parameters. $p_{k}^{F o r}$ (resp. $p_{k}^{D o m}$ ) indicates the term $\sigma_{k} / 2+\kappa_{k} / \sigma_{k}+\rho_{k} a_{k}^{F o r}$ (resp. $\sigma_{k} / 2+\kappa_{k} / \sigma_{k}+\rho_{k} a_{k}^{D o m}$ ) in (32) for the foreign (resp. domestic) currency. The corresponding benchmarked savings account is a true martingale if and only if these terms are non negative for all $k=1,2$. 


\begin{tabular}{cccc} 
Parameters & EURUSD & EURJPY & USDJPY \\
\hline$V_{1}$ & 1.641952 & 0.836130 & 1.717215 \\
$V_{2}$ & 0.439494 & 1.050021 & 0.745681 \\
$\kappa_{1}$ & 0.181556 & -0.680380 & 0.235865 \\
$\kappa_{2}$ & 0.10280 & 0.472494 & 0.643794 \\
$\sigma_{1}$ & 3.065151 & 2.010454 & 3.147204 \\
$\sigma_{2}$ & 0.794530 & 0.564713 & 1.228448 \\
$\theta_{1}$ & 0.028176 & -0.819852 & -0.013523 \\
$\theta_{2}$ & -0.218581 & 0.533048 & -0.330641 \\
$\rho_{1}$ & -0.87744 & -0.16380 & -0.155919 \\
$\rho_{2}$ & -0.43356 & -0.617496 & -0.944246 \\
$a_{1}^{\text {dom }}$ & 1.432145 & 1.813486 & 1.45799 \\
$a_{2}^{\text {dom }}$ & 1.533578 & 1.816593 & 1.394977 \\
$a_{1}^{\text {for }}$ & 1.422160 & 1.685392 & 1.375851 \\
$a_{2}^{\text {for }}$ & 1.403402 & 1.782033 & 1.19359 \\
Res. norm. & $3.45 \mathrm{e}-4$ & $2.05 \mathrm{e}-05$ & $2.77 \mathrm{e}-4$ \\
\hline$p_{1}^{\text {For }}$ & 0.343937 & 0.39072 & 1.434024 \\
$p_{2}^{\text {For }}$ & -0.081807 & 0.018654 & 0.011245 \\
$p_{1}^{\text {Dom }}$ & 0.335176 & 0.369745 & 1.421217 \\
$p_{2}^{\text {Dom }}$ & -0.13827 & -0.002685 & -0.178907 \\
\hline
\end{tabular}

Table 4: This table reports the results of the calibration of the model in the two factor case with market data as of 2nd May 2013. We include 4 maturities: 1 month, 3 months, 6 months, 1 year. For each column we show the calibrated parameters corresponding to a different pair of the triangle EUR/USD/JPY. We follow the convention FORDOM (e.g. EURUSD means that USD is the domestic currency, EUR is the foreign one). Strikes range in the set 10DP (i.e. 10 Delta Put), 25DP, ATM, 25DC, 10DC (for the market convention we refer to De Col et al. (2013)). The reference exchange rates are $S^{E U R U S D}(0)=1.3169, S^{E U R J P Y}(0)=128.205, S^{U S D J P Y}(0)=97.35$. Res. norm. is the residual of the objective function for the given set of parameters. $p_{k}^{F o r}$ (resp. $p_{k}^{D o m}$ ) indicates the term $\sigma_{k} / 2+\kappa_{k} / \sigma_{k}+\rho_{k} a_{k}^{F o r}$ (resp. $\sigma_{k} / 2+\kappa_{k} / \sigma_{k}+\rho_{k} a_{k}^{D o m}$ ) in (32) for the foreign (resp. domestic) currency. The corresponding benchmarked savings account is a true martingale if and only if these terms are non negative for all $k=1,2$. 
Vol Differences for EURUSD

\begin{tabular}{ccccc}
\hline & $1 \mathrm{~m}$ & $3 \mathrm{~m}$ & $6 \mathrm{~m}$ & $1 \mathrm{y}$ \\
\hline 10DP & -0.0065 & -0.0002 & -0.0015 & -0.0046 \\
25DP & -0.0067 & -0.0003 & 0.0005 & 0.0001 \\
ATM & -0.0038 & 0.0016 & 0.0028 & 0.0040 \\
25DC & 0.0023 & 0.0036 & 0.0026 & 0.0034 \\
10DC & 0.0089 & 0.0031 & -0.0011 & 0.0082 \\
\hline \hline Vol Differences for EURJPY & & & & \\
& & & & \\
\hline & $1 \mathrm{~m}$ & $3 \mathrm{~m}$ & $6 \mathrm{~m}$ & $1 \mathrm{y}$ \\
\hline 10DP & 0.0005 & 0.0004 & 0.0016 & -0.0000 \\
25DP & -0.0004 & -0.0018 & -0.0010 & -0.0017 \\
ATM & -0.0003 & -0.0004 & 0.0006 & 0.0016 \\
25DC & 0.0013 & 0.0010 & 0.0014 & -0.0004 \\
10DC & -0.0009 & -0.0002 & -0.0012 & 0.0002 \\
\hline \hline Vol Differences for USDJPY & & & & \\
& & & & \\
\hline 10DP & $1 \mathrm{~m}$ & $3 \mathrm{~m}$ & $6 \mathrm{~m}$ & $1 \mathrm{y}$ \\
25DP & -0.0040 & -0.0019 & 0.0036 & 0.0054 \\
ATM & -0.0049 & -0.0049 & -0.0022 & -0.0003 \\
25DC & -0.0045 & -0.0056 & -0.0039 & -0.0028 \\
10DC & 0.0002 & -0.0010 & -0.0001 & -0.0015 \\
\hline \hline & 0.0057 & 0.0048 & 0.0052 & -0.0016 \\
\hline \hline
\end{tabular}

Table 5: This table reports the differences between market implied volatilities and model implied volatilities in the two factor case with market data as of 2nd May 2013 corresponding to a different pair of the triangle EUR/USD/JPY. We include 4 maturities: 1 month, 3 months, 6 months, 1 year. We follow the convention FORDOM (e.g. EURUSD means that USD is the domestic currency, EUR is the foreign one). Strikes range in the set 10DP (i.e. 10 Delta Put), 25DP, ATM, 25DC, 10DC (for the market convention we refer to De Col et al. (2013)). The reference exchange rates are $S^{E U R U S D}(0)=1.3169, S^{E U R J P Y}(0)=128.205, S^{U S D J P Y}(0)=97.35$. 